\title{
Helicity amplitudes for QCD with massive quarks
}

\author{
Alexander Ochirov \\ ETH Zürich, Institut für Theoretische Physik, \\ Wolfgang-Pauli-Str. 27, 8093 Zürich, Switzerland \\ E-mail: aochirov@phys.ethz.ch
}

ABSTRACT: The novel massive spinor-helicity formalism of Arkani-Hamed, Huang and Huang provides an elegant way to calculate scattering amplitudes in quantum chromodynamics for arbitrary quark spin projections. In this note we compute two families of treelevel QCD amplitudes with one massive quark pair and $n-2$ gluons. The two cases include all gluons with identical helicity and one opposite-helicity gluon being color-adjacent to one of the quarks. Our results naturally incorporate the previously known amplitudes for both quark spins quantized along one of the gluonic momenta. In the all-multiplicity formulae presented here the spin quantization axes can be tuned at will, which includes the case of the definite-helicity quark states.

KEywords: Perturbative QCD, Scattering Amplitudes, Space-Time Symmetries

ARXIV EPRINT: 1802.06730 


\section{Contents}

1 Introduction 1

2 Spinor-helicity review 2

2.1 Massless Weyl spinors 3

2.2 Massive Weyl spinors 4

2.3 Dirac spinors and spin 5

$\begin{array}{llr}3 & \text { Four-point amplitudes } & 7\end{array}$

$\begin{array}{lll}4 & \text { All-multiplicity amplitudes } & 10\end{array}$

$\begin{array}{lll}4.1 & \text { All-plus amplitudes with two quarks } & 10\end{array}$

$\begin{array}{lll}4.2 & \text { One-minus amplitudes with two quarks } & 11\end{array}$

5 Checks 13

6 Summary and discussion $\quad 16$

$\begin{array}{ll}\text { A Massless spinor parametrizations } & 17\end{array}$

$\begin{array}{ll}\text { B Massive spinor parametrizations } & 17\end{array}$

\section{Introduction}

The recent advances in the analytic understanding of the scattering amplitudes are often believed to be specific to massless theories, preferably with supersymmetry. It is arguably due to the absence, until recently, of a fully satisfactory spinor-helicity formalism for massive particles. Of course, the massless spinor-helicity formalism [1-6] (popularized e.g. by ref. [7]) has been applied [8-10] to define massive Dirac spinors. However, that construction did not manage to dispel the notion of the on-shell amplitude methods being restricted to the massless case. Recently, however, Arkani-Hamed, Huang and Huang [11] have introduced a complete version of a massive spinor-helicity formalism and used it to reconsider an array of quantum field-theoretic results from the fully on-shell perspective.

This note is about how this massive formalism can be used in one field theory of interest - quantum chromodynamics with heavy quarks. For simplicity, here we only consider the amplitudes with one massive quark-antiquark pair, with the other particles being gluons of definite helicity. The main goals of this note are two-fold:

- We provide new all-multiplicity expressions, eqs. (4.1) and (4.8), for the $n$-point colorordered amplitudes with two quarks in case of all gluons of identical helicity and the case of one gluon of opposite helicity color-adjacent to one of the quarks. 
- We pay special attention to our conventions so that our results be consistent with the vast $\mathrm{QCD}$ literature. That involves flexible transitions between the presented massive formalism, its massless analogue recovered in the high-energy limit, the general Dirac spinors and their realization using the massless Weyl spinors.

In view of the second goal, in section 2 we review the spinor-helicity formalism in an effort to combine brevity with comprehensiveness. We illustrate the introduced methods in section 3, where we show two ways to derive a full color-dressed amplitude for fourparticle scattering (corresponding e.g. to non-abelian Compton scattering). We highlight the difference between the Feynman-diagrammatic approach and the on-shell construction, which deals solely with gauge-invariant quantities.

In section 4 we present and prove the aforementioned all-multiplicity amplitudes with two specific gluon-helicity configurations. For that we employ the Britto-Cachazo-FengWitten $(\mathrm{BCFW})$ on-shell recursion $[12,13]$. The spins of the quark and the antiquark remain unfixed throughout the calculations, which lets us specialize to the specific quarkspin projections considered previously [14] in the massless-spinor-based formalism [8-10]. Hence, in section 5, we give a simple dictionary (5.4) between the two descriptions and thus compare our results with the literature. It also shows that the new formalism easily incorporates the old one, the elegance of which suffered from the loss of the explicit littlegroup $\mathrm{SU}(2)$ symmetry.

We hope that this note will pave the way to more tree- and loop-level calculations in the newly complete spinor-helicity formalism [11], as outlined in section 6 .

\section{Spinor-helicity review}

It is well-known that particles are defined as irreducible unitary representations of the Poincare group $[15,16]$. Once the translation operator is diagonalized and the particles are labeled by their momentum $p^{\mu}$, one is left with the Lorentz $\mathrm{SO}(1,3)$ subgroup of the Poincare group. The remaining labels of a one-particle state turn out to belong to a representation of its little group. This subgroup of $\mathrm{SO}(1,3)$ is crucial for understanding spin. It is defined through the Lorentz transformations that preserve the momentum $p^{\mu}$ of the particle. It corresponds to $\mathrm{SO}(2)$ for massless states or to $\mathrm{SO}(3)$ for massive ones.

To include fermions into consideration, one must generalize to the universal covering group $\mathrm{SL}(2, \mathbb{C})$ of $\mathrm{SO}(1,3)$. The homomorphism between these two groups is implemented by the spinor maps

$$
p_{\alpha \dot{\beta}}=p_{\mu} \sigma_{\alpha \dot{\beta}}^{\mu}, \quad p^{\dot{\alpha} \beta}=p^{\mu} \bar{\sigma}_{\mu}^{\dot{\alpha} \beta} .
$$

The Pauli matrices ${ }^{1} \sigma^{\mu}=\left(1, \sigma^{1}, \sigma^{2}, \sigma^{3}\right)$ and $\bar{\sigma}^{\mu}=\left(1,-\sigma^{1},-\sigma^{2},-\sigma^{3}\right)$ here translate Lorentz transformations between the spinorial and vectorial languages:

$$
L_{\nu}^{\mu}=\frac{1}{2} \operatorname{tr}\left(\bar{\sigma}^{\mu} S \sigma_{\nu} S^{\dagger}\right): \quad p_{\alpha \dot{\delta}} \rightarrow S_{\alpha}{ }^{\beta} p_{\beta \dot{\gamma}}\left(S_{\delta}^{\gamma}\right)^{*} \quad \Rightarrow \quad p^{\mu} \rightarrow L_{\nu}^{\mu} p^{\nu},
$$

for $L \in \mathrm{SO}(1,3)$ and $S \in \mathrm{SL}(2, \mathbb{C})$. At the same time, the little groups for massless and massive particles are accordingly promoted to $\mathrm{U}(1)$ and $\mathrm{SU}(2)$.

\footnotetext{
${ }^{1}$ We use $\sigma^{0}=\left(\begin{array}{ll}1 & 0 \\ 0 & 1\end{array}\right), \sigma^{1}=\left(\begin{array}{ll}0 & 1 \\ 1 & 0\end{array}\right), \sigma^{2}=\left(\begin{array}{cc}0 & -i \\ i & 0\end{array}\right), \sigma^{3}=\left(\begin{array}{cc}1 & 0 \\ 0 & -1\end{array}\right)$, as well as $\epsilon^{\alpha \beta}=\left(\begin{array}{cc}0 & 1 \\ -1 & 0\end{array}\right)$ and $\epsilon_{\alpha \beta}=\left(\begin{array}{cc}0 & -1 \\ 1 & 0\end{array}\right)$.
} 
An important property of the $\mathrm{SL}(2, \mathbb{C})$ transformations (and hence the $\mathrm{SU}(2)$ ones) is that they preserve the antisymmetric form $\epsilon_{\alpha \beta}=-\epsilon^{\alpha \beta}$, i.e. the spinor product:

$$
S_{\alpha}^{\gamma} S_{\beta}{ }^{\delta} \epsilon_{\gamma \delta}=\frac{1}{2} \epsilon_{\gamma \delta}\left(\delta_{\alpha}^{\phi} \delta_{\beta}^{\psi}-\delta_{\beta}^{\phi} \delta_{\alpha}^{\psi}\right) S_{\phi}^{\gamma} S_{\psi}^{\delta}=\frac{1}{2} \epsilon_{\gamma \delta} \epsilon_{\alpha \beta} \epsilon^{\phi \psi} S_{\phi}^{\gamma} S_{\psi}^{\delta}=\epsilon_{\alpha \beta} \operatorname{det} S=\epsilon_{\alpha \beta}
$$

This form allows to raise and lower both the spinor and massive-little-group indices at will.

Now let us explore different spinor types one by one. The massless and massive Weyl spinors comprise the spinor-helicity formalism $[1-6,11]$, while the Dirac spinors are helpful to connect it to the more traditional approaches.

\subsection{Massless Weyl spinors}

In the massless case, the on-shell condition $p^{2}=\operatorname{det}\left\{p_{\alpha \dot{\beta}}\right\}=0$ means that the degenerate matrix $p_{\alpha \dot{\beta}}$ can be decomposed as a tensor product of two Weyl spinors. That decomposition can be written in various interchangeable ways using the spinor bra-ket notation:

$$
\begin{aligned}
& p_{\alpha \dot{\beta}}=\lambda_{p \alpha} \tilde{\lambda}_{p \dot{\beta}} \equiv|p\rangle_{\alpha}\left[\left.p\right|_{\dot{\beta}}\right. \\
& \not p=|p\rangle[p|+| p]\langle p| \\
& \left.p^{\dot{\alpha} \beta} \stackrel{\Uparrow}{=} \tilde{\lambda}_{p}^{\dot{\alpha}} \lambda_{p}^{\beta} \equiv \mid p\right]^{\dot{\alpha}}\left\langle\left. p\right|^{\beta}\right. \\
& \begin{aligned}
\Leftrightarrow \quad p^{\mu} & \stackrel{\mathbb{1}}{=} \\
= & \frac{1}{2} \lambda_{p}^{\alpha} \sigma_{\alpha \dot{\beta}}^{\mu} \tilde{\lambda}_{p}^{\dot{\beta}} \equiv \frac{1}{2}\left\langle p\left|\sigma^{\mu}\right| p\right] \\
p_{\mu} \stackrel{\mathbb{1}}{=} & \frac{1}{2} \tilde{\lambda}_{p \dot{\alpha}} \bar{\sigma}_{\mu}^{\dot{\alpha} \beta} \lambda_{p \beta} \equiv \frac{1}{2}\left[p\left|\bar{\sigma}_{\mu}\right| p\right\rangle .
\end{aligned}
\end{aligned}
$$

This notation fits the spinor products [1-6] particularly well:

$$
\langle p q\rangle \equiv \lambda_{p}^{\alpha} \lambda_{q \alpha}=\lambda_{p}^{\alpha} \epsilon_{\alpha \beta} \lambda_{q}^{\beta}, \quad[p q] \equiv \tilde{\lambda}_{p \dot{\alpha}} \tilde{\lambda}_{q}^{\dot{\alpha}}=\tilde{\lambda}_{p \dot{\alpha}} \epsilon^{\dot{\alpha} \dot{\beta}} \tilde{\lambda}_{q \dot{\beta}}, \quad\langle p q\rangle[q p]=2 p \cdot q .
$$

The Lorentz transformations (2.2) act on the Weyl spinors $\lambda_{p \alpha} \equiv|p\rangle_{\alpha}$ and $\left.\tilde{\lambda}_{p}^{\dot{\alpha}} \equiv \mid p\right]^{\dot{\alpha}}$ via $S \in \mathrm{SL}(2, \mathbb{C})$, but only up to the little-group U(1) rotations: ${ }^{2}$

$$
\begin{array}{ll}
\lambda_{p \alpha} \rightarrow S_{\alpha}^{\beta} \lambda_{p \beta}=e^{i \phi / 2} \lambda_{L p \alpha}, & \lambda_{p}^{\alpha} \rightarrow \lambda_{p}^{\beta}\left(S^{-1}\right)_{\beta}^{\alpha}=e^{i \phi / 2} \lambda_{L p}^{\alpha}, \\
\tilde{\lambda}_{p \dot{\alpha}} \rightarrow \tilde{\lambda}_{p \dot{\beta}}\left(S^{\dagger}\right)_{\dot{\alpha}}^{\dot{\beta}}=e^{-i \phi / 2} \tilde{\lambda}_{L p \dot{\alpha}}, & \tilde{\lambda}_{p}^{\dot{\alpha}} \rightarrow\left(S^{\dagger-1}\right)_{\dot{\beta}}^{\dot{\alpha}} \tilde{\lambda}_{p}^{\dot{\beta}}=e^{-i \phi / 2} \tilde{\lambda}_{L p}^{\dot{\alpha}} .
\end{array}
$$

These spinors also give us the building blocks for the polarization vectors of gauge bosons:

$$
\begin{array}{lll}
\varepsilon_{p+}^{\mu}=\frac{1}{\sqrt{2}} \frac{\left\langle q\left|\sigma^{\mu}\right| p\right]}{\langle q p\rangle} & \Leftrightarrow & \xi_{p}^{+}=\sqrt{2} \frac{|q\rangle[p|+| p]\langle q|}{\langle q p\rangle}, \\
\varepsilon_{p-}^{\mu}=-\frac{1}{\sqrt{2}} \frac{\left[q\left|\bar{\sigma}^{\mu}\right| p\right\rangle}{[q p]} & \Leftrightarrow & \not_{p}^{-}=-\sqrt{2} \frac{|p\rangle[q|+| q]\langle p|}{[q p]},
\end{array}
$$

where $q$ can be any null vector such that $|q\rangle \nsim|p\rangle$ and $\mid q] \nsim \mid p]$. Indeed, different reference vectors are equivalent up to a pure gauge, e.g.

$$
\varepsilon_{p+}^{\mu}\left(q^{\prime}\right)=\varepsilon_{p+}^{\mu}(q)+\frac{\sqrt{2}\left\langle q^{\prime} q\right\rangle p^{\mu}}{\left\langle q^{\prime} p\right\rangle\langle p q\rangle} .
$$

\footnotetext{
${ }^{2}$ In the case that the Lorentz transformation $L$ is a pure $\mathrm{SO}(2)$ rotation around the momentum axis $\hat{p}$ by the angle $\phi$, the little-group phases in eq. (2.6) are unambiguous and precisely equal to $\pm \phi / 2$.
} 
Now it is important to note that under a Lorentz transformation (2.6) the polarization vectors do not actually transform as proper vectors. For instance, comparing

$$
L_{\nu}^{\mu} \varepsilon_{p+}^{\nu}=\frac{\left\langle\lambda_{q} S^{-1}\left|\sigma^{\mu}\right| S^{\dagger-1} \tilde{\lambda}_{p}\right]}{\sqrt{2}\left\langle\lambda_{q} S^{-1} \mid S \lambda_{p}\right\rangle}=e^{-i \phi} \frac{\left\langle\lambda_{q} S^{-1}\left|\sigma^{\mu}\right| \tilde{\lambda}_{L p}\right]}{\sqrt{2}\left\langle\lambda_{q} S^{-1} \mid \lambda_{L p}\right\rangle} \quad \text { vs. } \quad \varepsilon_{L p+}^{\mu} \equiv \frac{\left\langle\lambda_{q}\left|\sigma^{\mu}\right| \tilde{\lambda}_{L p}\right]}{\sqrt{2}\left\langle\lambda_{q} \lambda_{L p}\right\rangle}
$$

we conclude that Lorentz transformations act as

$$
\varepsilon_{p \pm}^{\mu} \rightarrow L_{\nu}^{\mu} \varepsilon_{p \pm}^{\nu} \sim e^{\mp i \phi} \varepsilon_{L p \pm}^{\mu}
$$

only up to an additional term proportional to the new momentum $L_{\nu}^{\mu} p^{\nu}$. However, up to this caveat, this shows that these polarization vectors can be thought of as conversion coefficients between the off-shell Lorentz transformations and the corresponding on-shell little-group rotations [11]. A similar statement for the Weyl spinors is demonstrated by eq. (2.6) and is also true for the massive case, see eq. (2.13) below.

As a concrete realization of the Weyl spinors, one could use, for instance,

$$
\lambda_{p \alpha}=\sqrt{2 E}\left(\begin{array}{c}
-e^{-i \varphi} \sin \frac{\theta}{2} \\
\cos \frac{\theta}{2}
\end{array}\right), \quad \tilde{\lambda}_{p}^{\dot{\alpha}}=\sqrt{2 E}\left(\begin{array}{c}
\cos \frac{\theta}{2} \\
e^{i \varphi} \sin \frac{\theta}{2}
\end{array}\right),
$$

for a null momentum expressible as $p^{\mu}=E(1, \cos \varphi \sin \theta, \sin \varphi \sin \theta, \cos \theta)$. A more practical implementation is given in appendix A.

\subsection{Massive Weyl spinors}

For a nonzero mass $m$, we have a non-degenerate matrix $p_{\alpha \dot{\beta}}$ that satisfies $\operatorname{det}\left\{p_{\alpha \dot{\beta}}\right\}=m^{2}$. The Weyl spinors are then introduced [11] by expanding $p_{\alpha \dot{\beta}}$ in terms of two explicitly degenerate matrices $\lambda_{p \alpha}^{1} \tilde{\lambda}_{p \dot{\beta} 1}$ and $\lambda_{p \alpha} \tilde{\lambda}_{p \dot{\beta} 2}$ :

$$
\begin{array}{ccc}
p_{\alpha \dot{\beta}}=\lambda_{p \alpha}^{a} \tilde{\lambda}_{p \dot{\beta} a}=\lambda_{p \alpha}^{a} \epsilon_{a b} \tilde{\lambda}_{p \dot{\beta}}^{b}, & \operatorname{det}\left\{\lambda_{p \alpha}^{a}\right\}=m, & \operatorname{det}\left\{\tilde{\lambda}_{p \dot{\alpha}}^{a}\right\}=m, \\
p^{\dot{\alpha} \beta} \stackrel{\mathbb{1}}{=} \tilde{\lambda}_{p a}^{\dot{\alpha}} \lambda_{p}^{\beta a}=-\tilde{\lambda}_{p}^{\dot{\alpha} a} \epsilon_{a b} \lambda_{p}^{\beta b}, & \lambda_{\alpha a} \lambda^{\beta a} \stackrel{\Downarrow}{=} m \delta_{\alpha}^{\beta}, & \tilde{\lambda}^{\dot{\alpha} a} \tilde{\lambda}_{\dot{\beta} a} \stackrel{\Downarrow}{=} m \delta_{\dot{\beta}}^{\dot{\alpha}} .
\end{array}
$$

Here we have already indicated that the little-group indices $a, b=1,2$ are lowered and raised by the antisymmetric form $\epsilon_{a b}$, preserved by $\mathrm{SU}(2)$ rotations. Such little-group transformations follow from the action of the Lorentz group on these spinors:

$$
\begin{array}{ll}
\lambda_{p \alpha}^{a} \rightarrow S_{\alpha}^{\beta} \lambda_{p \beta}^{a}=\omega_{b}^{a} \lambda_{L p \alpha}^{b}, & \lambda_{p}^{\alpha a} \rightarrow \lambda_{p}^{\beta a}\left(S^{-1}\right)_{\beta}^{\alpha}=\omega_{b}^{a} \lambda_{L p}^{\alpha b}, \\
\tilde{\lambda}_{p \dot{\alpha}}^{a} \rightarrow \tilde{\lambda}_{p \dot{\beta}}^{a}\left(S^{\dagger}\right)_{\dot{\alpha}}^{\dot{\beta}}=\omega_{b}^{a}{ }_{b} \tilde{\lambda}_{L p \dot{\alpha}}{ }^{b}, & \tilde{\lambda}_{p}^{\dot{\alpha} a} \rightarrow\left(S^{\dagger-1}\right)_{\dot{\beta}}^{\dot{\alpha}} \tilde{\lambda}_{p}^{\dot{\beta} a}=\omega_{b}^{a}{ }_{b} \tilde{\lambda}_{L p}^{\dot{\alpha} b},
\end{array}
$$

where $\omega \in \mathrm{SU}(2)$ correspond to the $\mathrm{SO}(3)$ rotations in the rest frame of the massive particle momentum. These transformations are a massive analogue of eq. (2.6). Furthermore, the momentum decomposition (2.12) implies the two-dimensional version of the Dirac equation

$$
p^{\dot{\alpha} \alpha} \lambda_{p \alpha}^{a}=m \tilde{\lambda}_{p}^{\dot{\alpha} a}, \quad p_{\alpha \dot{\alpha}} \tilde{\lambda}_{p}^{\dot{\alpha} a}=m \lambda_{p \alpha}^{a} .
$$


For further convenience, let us rewrite the above identities in the spinor bra-ket notation:

$$
\begin{aligned}
& \left|p^{a}\right\rangle_{\alpha}\left[\left.p_{a}\right|_{\dot{\beta}}=p_{\alpha \dot{\beta}} \quad \quad p^{\dot{\alpha} \beta}\left|p^{a}\right\rangle_{\beta}=m \mid p^{a}\right]^{\dot{\alpha}} \\
& \left.\left.\mid p^{a}\right]^{\dot{\alpha}}\left\langle\left. p_{a}\right|^{\beta}=-p^{\dot{\alpha} \beta} \quad \quad p_{\alpha \dot{\beta}}\right| p^{a}\right]^{\dot{\beta}}=m\left|p^{a}\right\rangle_{\alpha} \quad\left\langle p^{a} p^{b}\right\rangle=-m \epsilon^{a b} \\
& \left|p^{a}\right\rangle_{\alpha}\left\langle\left. p_{a}\right|^{\beta}=-m \delta_{\alpha}^{\beta} \quad\left\langle\left. p^{a}\right|^{\alpha} p_{\alpha \dot{\beta}}=-m\left[\left.p^{a}\right|_{\dot{\beta}} \quad\left[p^{a} p^{b}\right]=m \epsilon^{a b} .\right.\right.\right. \\
& \left.\mid p^{a}\right]^{\dot{\alpha}}\left[\left.p_{a}\right|_{\dot{\beta}}=m \delta_{\dot{\beta}}^{\dot{\alpha}} \quad\left[\left.p^{a}\right|_{\dot{\alpha}} p^{\dot{\alpha} \beta}=-m\left\langle\left. p^{a}\right|^{\beta}\right.\right.\right.
\end{aligned}
$$

As an explicit spinor realization, one may use [11]

$\lambda_{p \alpha}^{a}=\left(\begin{array}{cc}\sqrt{E-P} \cos \frac{\theta}{2}-\sqrt{E+P} e^{-i \varphi} \sin \frac{\theta}{2} \\ \sqrt{E-P} e^{i \varphi} \sin \frac{\theta}{2} & \sqrt{E+P} \cos \frac{\theta}{2}\end{array}\right), \quad \tilde{\lambda}_{p \dot{\alpha}}^{a}=\left(\begin{array}{cc}-\sqrt{E+P} e^{i \varphi} \sin \frac{\theta}{2} & -\sqrt{E-P} \cos \frac{\theta}{2} \\ \sqrt{E+P} \cos \frac{\theta}{2} & -\sqrt{E-P} e^{-i \varphi} \sin \frac{\theta}{2}\end{array}\right)$,

given a massive momentum expressible as $p^{\mu}=(E, P \cos \varphi \sin \theta, P \sin \varphi \sin \theta, P \cos \theta)$, such that $E^{2}-P^{2}=m^{2}$. A more detailed implementation is given in appendix B.

\subsection{Dirac spinors and spin}

In this paper, we wish to study massive quarks that are traditionally described in terms of the Dirac spinors. Hence it may be illuminating to consider how the Weyl spinors (2.12) naturally unify into the Dirac spinors: ${ }^{3}$

$$
\begin{aligned}
& u_{p}^{a}=\left(\begin{array}{c}
\lambda_{p a}^{a} \\
\tilde{\lambda}_{p}^{\dot{\alpha} a}
\end{array}\right)=\left(\begin{array}{l}
\left|p^{a}\right\rangle_{\alpha} \\
\left.\mid p^{a}\right]^{\dot{\alpha}}
\end{array}\right) \\
& \bar{u}_{p}^{a}=\left(\begin{array}{r}
-\lambda_{p a}^{\alpha a} \\
\tilde{\lambda}_{p \dot{\alpha}}^{a}
\end{array}\right)=\left(\begin{array}{r}
-\left\langle\left. p^{a}\right|^{\alpha}\right. \\
{\left[\left.p^{a}\right|_{\dot{\alpha}}\right.}
\end{array}\right)
\end{aligned} \quad \Rightarrow \quad\left\{\begin{array}{l}
(\not p-m) u_{p}^{a}=\bar{u}_{p}^{a}(\not p-m)=0, \\
\bar{u}_{p}^{a} u_{p}^{b}=2 m \epsilon^{a b}, \\
\bar{u}_{p}^{a} \gamma^{\mu} u_{p}^{b}=2 p^{\mu} \epsilon^{a b}, \\
u_{p}^{a} \bar{u}_{p a}=u_{p}^{a} \epsilon_{a b} \bar{u}_{p}^{b}=\not p+m .
\end{array}\right.
$$

This choice of $\bar{u}_{p}^{a}$ is consistent with the conjugation property $\left(u_{p}^{a}\right)^{\dagger}=\operatorname{sgn}\left(p^{0}\right) \bar{u}_{p a} \gamma^{0}$. The energy sign here is due to our convention

$$
m=\operatorname{sgn}\left(p^{0}\right) \sqrt{p^{2}}
$$

which defines the signs of the energy and mass to be the same. As discussed in appendix B, this lets us cover the $u$ - and $v$-spinors together simultaneously by eq. (2.17).

We can treat these spinors as quantum-mechanical wavefunctions and compute the expectation values of the spin operator $\vec{\Sigma} / 2$, where $\Sigma^{i} \equiv i \epsilon^{i j k} \gamma_{j} \gamma_{k} / 2$. Given the spinor parametrization $(2.16)$, we obtain the three-dimensional spin vector

$$
\vec{s}\left(u_{p}^{a}\right) \equiv \frac{1}{2} \frac{u_{p}^{a \dagger} \vec{\Sigma} u_{p}^{a}}{u_{p}^{a \dagger} u_{p}^{a}}=\frac{\bar{u}_{p a} \vec{\gamma} \gamma^{5} u_{p}^{a}}{2 \bar{u}_{p a} \gamma^{0} u_{p}^{a}}=\frac{(-1)^{a-1}}{2}(\cos \varphi \sin \theta, \sin \varphi \sin \theta, \cos \theta) \equiv \frac{(-1)^{a-1}}{2} \hat{p} .
$$

Therefore, the spinors (2.16) have definite $\pm 1 / 2$ helicities, i.e. the eigenvalues of the helicity operator $h=\hat{p} \cdot \vec{\Sigma} / 2$, which is a conserved quantity for a one-particle state.

\footnotetext{
${ }^{3}$ We use the Dirac matrices in the Weyl basis, $\gamma^{\mu}=\left(\begin{array}{cc}0 & \sigma^{\mu} \\ \bar{\sigma}^{\mu} & 0\end{array}\right)$, hence $\gamma^{5}=\left(\begin{array}{cc}-1 & 0 \\ 0 & 1\end{array}\right)$ and $\vec{\Sigma}=\gamma^{0} \vec{\gamma} \gamma^{5}=\left(\begin{array}{cc}\vec{\sigma} & 0 \\ 0 & \vec{\sigma}\end{array}\right)$.
} 
To delve into the subject of spin a bit further, we rewrite the massive spinor parametrization $(2.16)$ as

$$
\begin{aligned}
& \lambda_{p \alpha}^{a}=\sqrt{E+P}\left(\begin{array}{c}
-e^{-i \varphi} \sin \frac{\theta}{2} \\
\cos \frac{\theta}{2}
\end{array}\right)_{\alpha} \otimes\left(\begin{array}{l}
0 \\
1
\end{array}\right)^{a}+\frac{m}{\sqrt{E+P}}\left(\begin{array}{c}
\cos \frac{\theta}{2} \\
e^{i \varphi} \sin \frac{\theta}{2}
\end{array}\right)_{\alpha} \otimes\left(\begin{array}{l}
1 \\
0
\end{array}\right)^{a}, \\
& \tilde{\lambda}_{p}^{\dot{\alpha} a}=\sqrt{E+P}\left(\begin{array}{c}
\cos \frac{\theta}{2} \\
e^{i \varphi} \sin \frac{\theta}{2}
\end{array}\right)^{\dot{\alpha}} \otimes\left(\begin{array}{l}
1 \\
0
\end{array}\right)^{a}+\frac{m}{\sqrt{E+P}}\left(\begin{array}{c}
-e^{-i \varphi} \sin \frac{\theta}{2} \\
\cos \frac{\theta}{2}
\end{array}\right)^{\dot{\alpha}} \otimes\left(\begin{array}{l}
0 \\
1
\end{array}\right)^{a},
\end{aligned}
$$

which makes obvious the smooth limit of the massive spinors $\lambda_{p \alpha}$ and $\tilde{\lambda}_{p}^{\dot{\alpha}}$ to their massless homonymes (2.11):

$$
\lambda_{p \alpha}^{a} \underset{m \rightarrow 0}{\longrightarrow} \lambda_{p \alpha} \zeta_{-}^{a}, \quad \tilde{\lambda}_{p}^{\dot{\alpha} a} \underset{m \rightarrow 0}{\longrightarrow} \tilde{\lambda}_{p}^{\dot{\alpha}} \zeta_{+}^{a},
$$

where $\zeta_{-}^{a} \equiv(0,1)$ and $\zeta_{+}^{a} \equiv(1,0)$. To rephrase this in a more general way, we can introduce two-dimensional spinors $\lambda_{p \alpha}$ and $\eta_{p \alpha}$ such that $\lambda_{p \alpha}^{a}$ and $\tilde{\lambda}_{p}^{\dot{\alpha} a}$ decompose as

$$
\lambda_{p \alpha}^{a}=\lambda_{p \alpha} \zeta_{-}^{a}+\eta_{p \alpha} \zeta_{+}^{a}, \quad \tilde{\lambda}_{p}^{\dot{\alpha} a}=\tilde{\lambda}_{p}^{\dot{\alpha}} \zeta_{+}^{a}-\tilde{\eta}_{p}^{\dot{\alpha}} \zeta_{-}^{a}, \quad\left\langle\lambda_{p} \eta_{p}\right\rangle=\left[\tilde{\eta}_{p} \tilde{\lambda}_{p}\right]=m .
$$

The massive momentum is now expressed as a sum of two null momenta:

$$
p_{\alpha \dot{\alpha}}=\lambda_{p \alpha} \tilde{\lambda}_{p \dot{\alpha}}+\eta_{p \alpha} \tilde{\eta}_{p \dot{\alpha}}
$$

which gives a link to the massive extension of the massless spinor-helicity formalism used previously in the literature [8-10]. We make this link precise in section 5 below.

Now let us discuss a subtle point concerning spin. Traditional quantum-mechanical spin operators are thought of as acting on the $\mathrm{SU}(2)$ indices, which correspond to the little group. The spin of the decomposition (2.16) points along the three-momentum $\vec{p}$, whereas the little-group vectors $\zeta_{ \pm}^{a}$ seem to describe states with spin direction along the $z$ axis. In other words, the massive Weyl spinors (2.16) convert the physical helicity operator $h=\hat{p} \cdot \vec{\Sigma} / 2=\left(\begin{array}{cc}\hat{p} \cdot \vec{\sigma} & 0 \\ 0 & \hat{p} \cdot \vec{\sigma}\end{array}\right) / 2$ to $\sigma^{3} / 2$ :

$$
(\hat{p} \cdot \vec{\sigma})_{\alpha}^{\beta} \lambda_{p \beta}^{a}=\sigma_{b}^{3 a}{ }_{b}{ }_{p \alpha}^{b}, \quad(\hat{p} \cdot \vec{\sigma})_{\dot{\beta}}^{\dot{\alpha}} \tilde{\lambda}_{p}^{\dot{\beta} a}=\sigma_{b}^{3 a} \tilde{\lambda}_{p}^{\dot{\alpha} b} .
$$

This should be regarded as a nice feature of the parametrization (2.16) rather than an inconsistency. Indeed, the little-group $\mathrm{SU}(2)$ transformations correspond to $\mathrm{SO}(3)$ rotations in the rest frame of the massive particle, in which $p_{\text {rest }}^{\mu}=(m, \overrightarrow{0})$, whereas the spinorial matrices $\vec{\sigma}_{\alpha}^{\beta}=\vec{\sigma}_{\dot{\beta}}^{\dot{\alpha}}$ generate rotations in the boosted frame where $p^{\mu}=(E, \vec{p})$. It is therefore convenient that the spinorial $(\hat{p} \cdot \vec{\sigma}),{ }^{4}$ taken along the momentum direction, are converted to the simplest of the Pauli matrices, $\sigma^{3 a}{ }_{b}$.

In principle, one can easily break the above property by $\mathrm{SU}(2)$-rotating the spin states. Apart from losing the relatively simple parametrization (2.16), this would mix the pure

\footnotetext{
${ }^{4}$ In fact, the other two spatial directions corresponding to the little-group matrices $\sigma_{b}^{1 a}$ and $\sigma^{2 a}{ }_{b}$ in the sense of eq. (2.24) turn out to be complex for any nonzero $\vec{p}$. The corresponding spin-projection operators are thus not hermitian, and there is no unitary intertwining operator between the two representations of the complete spin operator $\vec{\sigma} / 2$. Indeed, such an operator would have to involve a boost transformation to the rest frame, which lies outside the rotational $\mathrm{SU}(2)$.
} 
helicity eigenstates and produce wavefunctions with a spin quantization axis other than the momentum, and therefore undetermined helicity. The massive spinor-helicity formalism of ref. [11] reviewed here allows to easily switch that axis, and this is precisely what we do in section 5 in order to compare our results with the literature.

\section{Four-point amplitudes}

In this section, we demonstrate the use of the various spinors discussed above by dissecting one full color-dressed amplitude. It is convenient to consider the simple case of one massive quark-antiquark pair and two gluons of opposite helicity. Their scattering amplitude has three Feynman diagrams: ${ }^{5}$

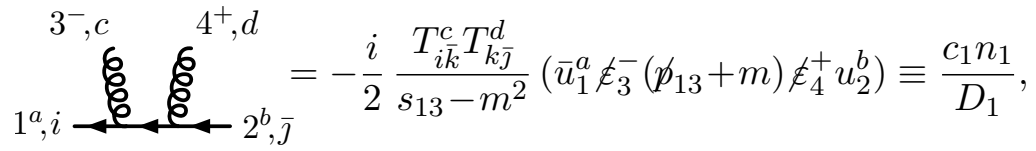

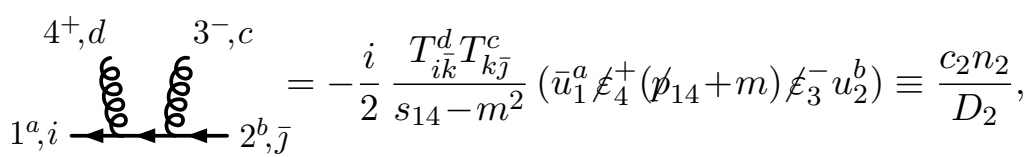

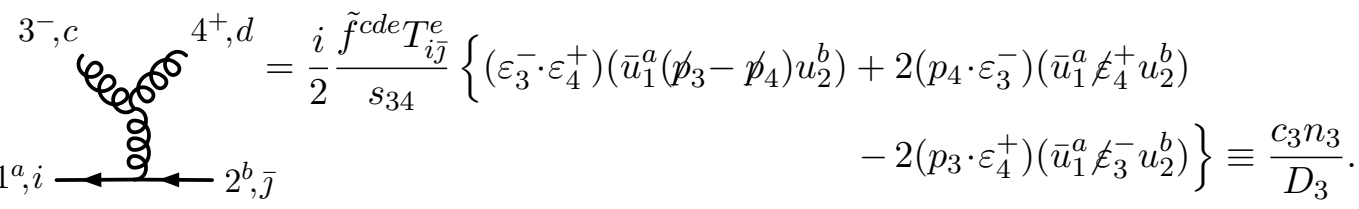

Here $u_{2}^{b}$ denotes the Dirac spinor corresponding to negative energy and mass $m_{2}=-m_{1}=$ $-m$. It is our energy sign convention (2.18) that allows us not to use a separate spinor $v_{2}^{b}$ for $u_{2}^{b}$. Now let us recast the above numerators in the spinor-helicity formalism by plugging in the Dirac spinors (2.17) and the polarization vectors (2.7),

$$
\begin{aligned}
n_{1}=\frac{-i}{\left[3 q_{3}\right]\left\langle 4 q_{4}\right\rangle}\{ & \left\langle\underline{1}^{a} 3\right\rangle\left[q_{3}\left|p_{13}\right| q_{4}\right\rangle\left[4 \overline{2}^{b}\right]-\left[\underline{1}^{a} q_{3}\right]\left\langle 3\left|p_{13}\right| 4\right]\left\langle q_{4} \overline{2}^{b}\right\rangle \\
& \left.+m\left\langle\underline{1}^{a} 3\right\rangle\left[q_{3} 4\right]\left\langle q_{4} \overline{2}^{b}\right\rangle-m\left[\underline{1}^{a} q_{3}\right]\left\langle 3 q_{4}\right\rangle\left[4 \overline{2}^{b}\right]\right\}, \\
n_{2}=\frac{-i}{\left[3 q_{3}\right]\left\langle 4 q_{4}\right\rangle}\{ & \left\langle\underline{1}^{a} q_{4}\right\rangle\left[4\left|p_{14}\right| 3\right\rangle\left[q_{3} \overline{2}^{b}\right]-\left[\underline{1}^{a} 4\right]\left\langle q_{4}\left|p_{14}\right| q_{3}\right]\left\langle 3 \overline{2}^{b}\right\rangle \\
& \left.+m\left\langle\underline{1}^{a} q_{4}\right\rangle\left[4 q_{3}\right]\left\langle 3 \overline{2}^{b}\right\rangle-m\left[\underline{1}^{a} 4\right]\left\langle q_{4} 3\right\rangle\left[q_{3} \overline{2}^{b}\right]\right\}, \\
n_{3}=\frac{-i}{\left[3 q_{3}\right]\left\langle 4 q_{4}\right\rangle}\{ & -{ }_{2}^{2}\left\langle 3 q_{4}\right\rangle\left[4 q_{3}\right]\left(\left\langle\underline{1}^{a}\left|p_{3}-p_{4}\right| \overline{2}^{b}\right]-\left[\underline{1}^{a}\left|p_{3}-p_{4}\right| \overline{2}^{b}\right\rangle\right) \\
& \left.-\left\langle 3|4| q_{3}\right]\left(\left\langle\underline{1}^{a} q_{4}\right\rangle\left[4 \overline{2}^{b}\right]-\left[\underline{1}^{a} 4\right]\left\langle q_{4} \overline{2}^{b}\right\rangle\right)+\left\langle q_{4}|3| 4\right]\left(\left\langle\underline{1}^{a} 3\right\rangle\left[q_{3} \overline{2}^{b}\right]-\left[\underline{1}^{a} q_{3}\right]\left\langle 3 \overline{2}^{b}\right\rangle\right)\right\},
\end{aligned}
$$

where for brevity we label spinors as $|i\rangle \equiv\left|p_{i}\right\rangle$, etc. We also underline the massive positiveenergy spinors and overline the negative-energy ones. The numerators (3.2) may seem

\footnotetext{
${ }^{5}$ We normalize the group generators to obey $\operatorname{Tr}\left(T^{a} T^{b}\right)=\delta^{a b}$ and $\left[T^{a}, T^{b}\right]=\tilde{f}^{a b c} T^{c}$ and regard all particle momenta as outgoing. We use slashed matrices $\not p$ to denote either $\gamma^{\mu} p_{\mu}, \sigma^{\mu} p_{\mu}$ or $\bar{\sigma}^{\mu} p_{\mu}$, depending on the spinors surrounding them. In expressions like $\langle i|j| k] \equiv\left\langle i\left|\not p_{j}\right| k\right]=\left\langle i\left|p_{j}\right| k\right]$ the slash can be omitted.
} 
complicated, which is due to their explicit gauge dependence on the gluonic reference vectors $q_{3}$ and $q_{4}$. Incidentally, one can check that for any such gauge choice they nontrivially satisfy the kinematic-algebra relation $n_{1}-n_{2}=n_{3}$, which is color-dual to the commutation relation $c_{1}-c_{2}=c_{3}[17,18]$. A very beneficial gauge choice is $q_{3}=p_{4}$ and $q_{4}=p_{3}$, for which

$$
n_{1}=n_{2}=\frac{i}{s_{34}}\langle 3|1| 4]\left(\left\langle\underline{1}^{a} 3\right\rangle\left[\overline{2}^{b} 4\right]-\left[\underline{1}^{a} 4\right]\left\langle\overline{2}^{b} 3\right\rangle\right), \quad n_{3}=0 .
$$

We can thus write simple closed-form expressions for all three color-ordered amplitudes

$$
\begin{aligned}
& A\left(\underline{1}^{a}, \overline{2}^{b}, 3^{-}, 4^{+}\right) \equiv \frac{n_{2}}{D_{2}}-\frac{n_{3}}{D_{3}}=\frac{i\langle 3|1| 4]}{\left(s_{14}-m^{2}\right) s_{34}}\left(\left\langle\underline{1}^{a} 3\right\rangle\left[\overline{2}^{b} 4\right]-\left[\underline{1}^{a} 4\right]\left\langle\overline{2}^{b} 3\right\rangle\right), \\
& A\left(\underline{1}^{a}, \overline{2}^{b}, 4^{+}, 3^{-}\right) \equiv \frac{n_{1}}{D_{1}}+\frac{n_{3}}{D_{3}}=\frac{i\langle 3|1| 4]}{\left(s_{13}-m^{2}\right) s_{34}}\left(\left\langle\underline{1}^{a} 3\right\rangle\left[\overline{2}^{b} 4\right]-\left[\underline{1}^{a} 4\right]\left\langle\overline{2}^{b} 3\right\rangle\right), \\
& A\left(\underline{1}^{a}, 3^{-}, \overline{2}^{b}, 4^{+}\right) \equiv-\frac{n_{1}}{D_{1}}-\frac{n_{2}}{D_{2}}=\frac{i\langle 3|1| 4]}{\left(s_{13}-m^{2}\right)\left(s_{14}-m^{2}\right)}\left(\left\langle\underline{1}^{a} 3\right\rangle\left[\overline{2}^{b} 4\right]-\left[\underline{1}^{a} 4\right]\left\langle\overline{2}^{b} 3\right\rangle\right) .
\end{aligned}
$$

These evidently obey the Kleiss-Kuijf relation $A_{1243}+A_{1234}+A_{1324}=0$ [19], as well as the Bern-Carrasco-Johansson (BCJ) relation [18, 20, 21]

$$
\left(s_{14}-m^{2}\right) A\left(\underline{1}^{a}, \overline{2}^{b}, 3^{-}, 4^{+}\right)=\left(s_{13}-m^{2}\right) A\left(\underline{1}^{a}, \overline{2}^{b}, 4^{+}, 3^{-}\right) .
$$

The full color-dressed amplitude can thus be constructed from a single linearly independent color-ordered amplitude as [18, 22]

$$
\mathcal{A}\left(1_{i}^{a}, 2_{\bar{\jmath}}^{b}, 3_{c}^{-}, 4_{d}^{+}\right)=i\left[\frac{T_{i \bar{k}}^{c} T_{k \bar{\jmath}}^{d}}{\left(s_{13}-m^{2}\right) s_{34}}+\frac{T_{i \bar{k}}^{d} T_{k \bar{j}}^{c}}{\left(s_{14}-m^{2}\right) s_{34}}\right]\langle 3|1| 4]\left(\left\langle\underline{1}^{a} 3\right\rangle\left[\overline{2}^{b} 4\right]-\left[\underline{1}^{a} 4\right]\left\langle\overline{2}^{b} 3\right\rangle\right) .
$$

It is interesting to note [23] that the gluonic color-ordered amplitude (3.4c) is also the correct QED amplitude [11] (up to a factor of -2 due to the color-generator conventions).

Note that the above amplitudes are gauge-invariant and could have been reduced from the numerators (3.2) to the expressions (3.4) for any choice of reference vectors $q_{3}$ and $q_{4}$. This illustrates why in general, at least in analytic calculations, it is better to avoid dealing with gauge-dependent objects and compute gauge-invariant quantities directly. Such a way to derive the above amplitudes would be via the BCFW on-shell recursion $[12,13]$ starting from the three-point amplitudes

$$
\left.\begin{array}{l}
\mathcal{A}\left(1_{i}^{a}, 2_{\bar{\jmath}}^{b}, 3_{c}^{+}\right)=-\frac{i T_{i \bar{\jmath}}^{c}}{\langle 3 q\rangle}\left(\left\langle\underline{1}^{a} q\right\rangle\left[\overline{2}^{b} 3\right]-\left[\underline{1}^{a} 3\right]\left\langle\overline{2}^{b} q\right\rangle\right)=i T_{i \bar{\jmath}^{c}} \frac{\left\langle\underline{1}^{a} \overline{2}^{b}\right\rangle[3|1| q\rangle}{m\langle 3 q\rangle} \\
\mathcal{A}\left(1_{i}^{a}, 2_{\bar{\jmath}}^{b}, 3_{c}^{-}\right)=\frac{i T_{i \bar{\jmath}}^{c}}{[3 q]}\left(\left\langle\underline{1}^{a} 3\right\rangle\left[\overline{2}^{b} q\right]-\left[\underline{1}^{a} q\right]\left\langle\overline{2}^{b} 3\right\rangle\right)=i T_{i \bar{\jmath}}^{c} \frac{\left[\underline{1}^{a} \overline{2}^{b}\right]\langle 3|1| q]}{m[3 q]}
\end{array}\right\}=\begin{gathered}
3^{ \pm}, c \\
1^{a}, i
\end{gathered}
$$

These make sense on complex on-shell kinematics and are independent of the gauge-boson reference vector $q$, despite not looking that way (this feature is explained in ref. [11]).

To reduce the four-point amplitude to the three-point ones, we apply a simple masslessspinor shift

$$
\mid \hat{3}] \equiv \mid 3]-z \mid 4], \quad|\hat{4}\rangle \equiv|4\rangle+z|3\rangle,
$$




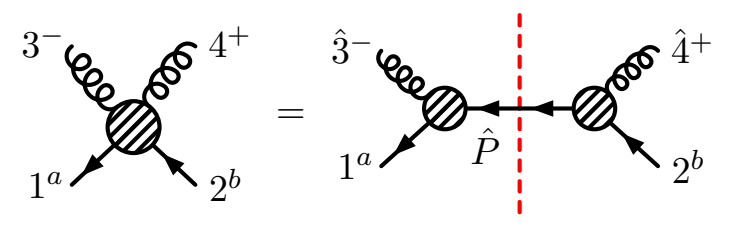

Figure 1. Graphic representation for the BCFW derivation (3.9) of $A\left(\underline{1}^{a}, \hat{3}^{-}, \hat{4}^{+}, \overline{2}^{b}\right)$.

which preserves momentum conservation and the on-shell conditions for any complex $z$. Cauchy's integral theorem then localizes the four-point amplitude $A\left(\underline{1}^{a}, \overline{2}^{b}, 4^{+}, 3^{-}\right)$on the only pole $z_{13}=\langle 3|1| 3] /\langle 3|1| 4]$ corresponding to the quark propagator $\hat{P} \equiv p_{1}+\hat{p}_{3}$, see figure 1. Hence the amplitude factorizes as a product of two three-point amplitudes with complex momenta:

$$
\begin{aligned}
& A\left(\underline{1}^{a}, \overline{2}^{b}, 4^{+}, 3^{-}\right)=\operatorname{Res}_{z=z_{13}} A\left(\underline{1}^{a}, \hat{3}^{-}, \hat{4}^{+}, \overline{2}^{b}\right)=A\left(\underline{1}^{a}, \hat{3}^{-},-\hat{P}^{c}\right) \frac{1}{s_{13}-m^{2}} A\left(\hat{P}_{c}, \hat{4}^{+}, \overline{2}^{b}\right) \\
&= \frac{1}{\left(s_{13}-m^{2}\right)[\hat{3} q 3]\left\langle\hat{4} q_{4}\right\rangle}\left(\left\langle\underline{1}^{a} 3\right\rangle\left[-\hat{P}^{c} \mid q_{3}\right]-\left[\underline{1}^{a} q_{3}\right]\left\langle-\hat{P}^{c} \mid 3\right\rangle\right)\left(\left\langle\hat{P}_{c} q_{4}\right\rangle\left[\overline{2}^{b} 4\right]-\left[\hat{P}_{c} 4\right]\left\langle\overline{2}^{b} q_{4}\right\rangle\right) \\
&= \frac{-i}{\left(s_{13}-m^{2}\right)[34]\langle 43\rangle}\left(\left\langle\underline{1}^{a} 3\right\rangle\left[4 \hat{P}^{c}\right]-\left[\underline{1}^{a} 4\right]\left\langle 3 \hat{P}^{c}\right\rangle\right)\left(\left\langle\hat{P}_{c} 3\right\rangle\left[\overline{2}^{b} 4\right]-\left[\hat{P}_{c} 4\right]\left\langle\overline{2}^{b} 3\right\rangle\right) \\
&= \frac{-i}{\left(s_{13}-m^{2}\right) s_{34}}\left\{\left\langle\underline{1}^{a} 3\right\rangle\left[\overline{2}^{b} 4\right]\left[4 \hat{P}^{c}\right]\left\langle\hat{P}_{c} 3\right\rangle-\left\langle\underline{1}^{a} 3\right\rangle\left\langle\overline{2}^{b} 3\right\rangle\left[4 \hat{P}^{c}\right]\left[\hat{P}_{c} 4\right]\right. \\
&=\left.\frac{i\langle 3|1| 4]}{\left(s_{13}-m^{2}\right) s_{34}}\left(\left\langle\underline{1}^{a} 3\right\rangle\left[\underline{2}^{b} 4\right]-\left[\overline{2}^{b} 4\right]\left\langle 3 \hat{P}^{c}\right\rangle\left\langle\hat{P}_{c}^{a} 3\right\rangle+\left[\underline{1}^{a} 4\right]\left\langle\overline{2}^{b} 3\right\rangle\left\langle 3 \hat{P}^{c}\right\rangle\left[\hat{P}_{c} 4\right]\right\}\right) .
\end{aligned}
$$

Here we chose the reference vectors as $q_{3}=p_{4}, q_{4}=p_{3}$ to remove most $z$-dependence as early as possible. Otherwise, the spinor products of $q_{3}$ and $q_{4}$ would cancel anyway, but only after plugging in the specific on-shell solutions for $\mid \hat{3}],|\hat{4}\rangle$ and $\hat{P}^{\mu}$ and using various Schouten identities. In the last transition of eq. (3.9), we also reduced the sum over the spin label $c$ of the intermediate quark using the completeness relations $(2.15){ }^{6}$

As a simple check, we verify that the massless limit corresponds to the well-known Parke-Taylor MHV amplitudes [24]:

$$
\mathcal{A}\left(1_{i}^{a}, 2_{\bar{\jmath}}^{b}, 3_{c}^{-}, 4_{d}^{+}\right) \underset{m \rightarrow 0}{\longrightarrow}-i\left[\frac{T_{i \bar{k}}^{c} T_{k \bar{\jmath}}^{d}}{\langle 12\rangle\langle 24\rangle\langle 43\rangle\langle 31\rangle}+\frac{T_{i \bar{k}}^{d} T_{k \bar{\jmath}}^{c}}{\langle 12\rangle\langle 23\rangle\langle 34\rangle\langle 41\rangle}\right]\left(\begin{array}{cc}
0 & \langle 13\rangle\langle 23\rangle^{3} \\
\langle 13\rangle^{3}\langle 23\rangle & 0
\end{array}\right) .
$$

It is even simpler calculation, either with the Feynman diagrams or via the on-shell recursion, to find the amplitudes with two quarks and two positive-helicity gluons

$$
A_{1234}=\frac{-i m\left\langle\underline{1}^{a} \overline{2}^{b}\right\rangle[34]}{\left(s_{14}-m^{2}\right)\langle 34\rangle}, \quad A_{1243}=\frac{-i m\left\langle\underline{1}^{a} \overline{2}^{b}\right\rangle[34]}{\left(s_{13}-m^{2}\right)\langle 34\rangle}, \quad A_{1324}=\frac{i m\left\langle\underline{1}^{a} \overline{2}^{b}\right\rangle[34]^{2}}{\left(s_{13}-m^{2}\right)\left(s_{14}-m^{2}\right)} .
$$

\footnotetext{
${ }^{6}$ Due to our mass sign convention (2.18), the quark masses are $m_{1}=m$ and $m_{2}=-m$, which is important when using the identities (2.15). The mass of the intermediate momentum along the fermion arrow is $m$.
} 


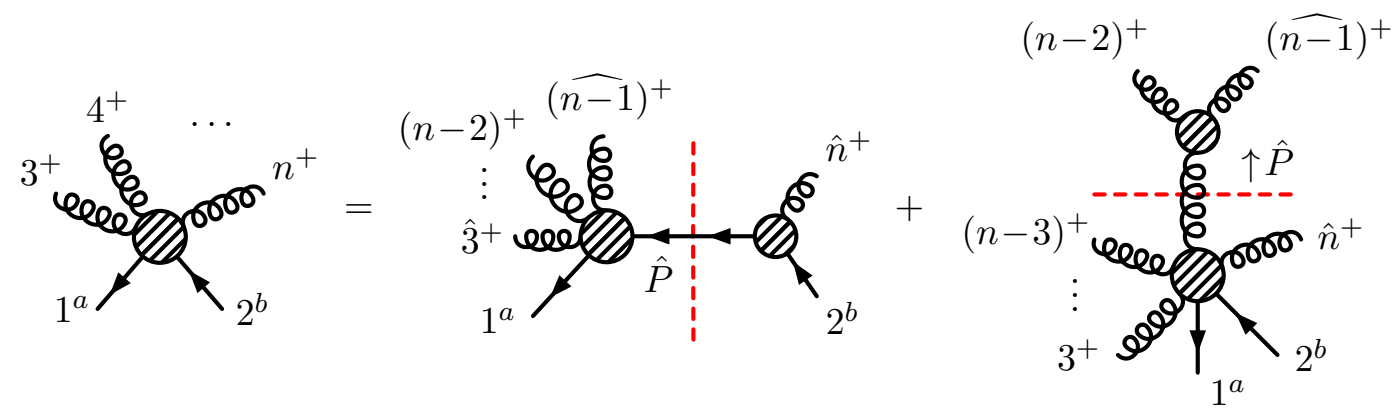

Figure 2. Graphic representation of the BCFW recursion step (4.3) for $A\left(\underline{1}^{a}, 3^{+}, 4^{+}, \ldots, n^{+}, \overline{2}^{b}\right)$.

\section{All-multiplicity amplitudes}

In this section we turn to the main calculations of this note - two infinite families of color-ordered amplitudes with one massive quark-antiquark pair.

\subsection{All-plus amplitudes with two quarks}

The $n$-point amplitude for a quark-antiquark pair and $n-2$ positive-helicity gluons equals

$$
A\left(\underline{1}^{a}, 3^{+}, 4^{+}, \ldots, n^{+}, \overline{2}^{b}\right)=\frac{-i m\left\langle\underline{1}^{a} \overline{2}^{b}\right\rangle\left[3\left|\prod_{j=3}^{n-2}\left\{\not p_{13 \ldots j} \not p_{j+1}+\left(s_{13 \ldots j}-m^{2}\right)\right\}\right| n\right]}{\left(s_{13}-m^{2}\right)\left(s_{134}-m^{2}\right) \ldots\left(s_{13 \ldots(n-1)}-m^{2}\right)\langle 34\rangle\langle 45\rangle \ldots\langle n-1 \mid n\rangle}
$$

It is easiest derived using the BCFW recursion [12, 13]. To set up the induction, we check that for $n=4$ the formula (4.1) visibly reduces to the four-point amplitude $A_{1243}$ in eq. (3.11). For the inductive step, we choose to shift the gluonic spinors

$$
\widehat{\mid n-1}] \equiv \mid n-1]-z \mid n], \quad|\hat{n}\rangle \equiv|n\rangle+z|n-1\rangle .
$$

Then well-known arguments $[14,25,26]$ guarantee a vanishing boundary behavior at $z \rightarrow$ $\infty$. There are two potential contributions in the on-shell recursion:

$$
\begin{array}{r}
A\left(\underline{1}^{a}, 3^{+}, 4^{+}, \ldots, n^{+}, \overline{2}^{b}\right)=A\left(\underline{1}^{a}, 3^{+}, \ldots,(n-2)^{+},(\widehat{n-1})^{+},-\hat{P}^{c}\right) \frac{1}{s_{2 n}-m^{2}} A\left(\hat{P}_{c}, \hat{n}^{+}, \overline{2}^{b}\right) \\
+A\left((n-2)^{+},(\widehat{n-1})^{+},-\hat{P}^{-}\right) \frac{i}{s_{(n-2)(n-1)}} A\left(\underline{1}^{a}, 3^{+}, \ldots,(n-3)^{+}, \hat{P}^{+}, \hat{n}^{+}, \overline{2}^{b}\right) .
\end{array}
$$

Any other $z$-dependent propagator would factorize on a vanishing purely gluonic amplitude with all positive helicities. The second pole in eq. (4.3) is localized on

$$
\left.\hat{s}_{(n-2)(n-1)}=0 \quad \Rightarrow \quad z=\frac{[n-2 \mid n-1]}{[n-2 \mid n]} \quad \Rightarrow \quad \widehat{n-1]}=\mid n-2\right] \frac{[n-1 \mid n]}{[n-2 \mid n]}
$$


which sets the three-point $\overline{\mathrm{MHV}}$ amplitude to zero. We then compute the remaining contribution on $z_{2 n}=-\langle n|2| n] /\langle n-1|2| n]$ :

$$
\begin{aligned}
A\left(\underline{1}^{a}, 3^{+}, \ldots, n^{+}, \overline{2}^{b}\right) & =\frac{\left\langle\underline{1}^{a} \mid-\hat{P}^{c}\right\rangle\left[3\left|\prod_{j=3}^{n-3}\left\{\not p_{13 \ldots j} \not p_{j+1}+\left(s_{13 \ldots j}-m^{2}\right)\right\}\right| \widehat{n-1}\right]\left\langle\hat{P}_{c} \overline{2}^{b}\right\rangle[n|\hat{P}| q\rangle}{\left(s_{13}-m^{2}\right) \ldots\left(s_{13 \ldots(n-2)}-m^{2}\right)\left(s_{2 n}-m^{2}\right)\langle 34\rangle \ldots\langle n-2 \mid n-1\rangle\langle\hat{n} q\rangle} \\
& =-\frac{i m\left\langle\underline{1}^{a} \overline{2}^{b}\right\rangle\left[3\left|\prod_{j=3}^{n-3}\left\{\not p_{13 \ldots j} \not \not_{j+1}+\left(s_{13 \ldots j}-m^{2}\right)\right\}\right| \widehat{n-1}\right]\langle n-1|2| n]}{\left(s_{13}-m^{2}\right) \ldots\left(s_{13 \ldots(n-1)}-m^{2}\right)\langle 34\rangle \ldots\langle n-2 \mid n-1\rangle\langle n-1 \mid n\rangle},
\end{aligned}
$$

where we picked $q=p_{n-1}$ to remove most of the $z$-dependence. To finish the proof of the closed-form expression (4.1), it now suffices to notice that

$$
\left.\left.\widehat{\mid n-1}]\langle n-1|2| n]=\not p_{(n-1) n} \not p_{2} \mid n\right]=\left\{\not p_{13 \ldots(n-2)} \not p_{n-1}+\left(s_{13 \ldots(n-2)}-m^{2}\right)\right\} \mid n\right] .
$$

It is effortless to also write the amplitude for the gluons of negative helicity: we simply exchange the angle and square brackets as in $\langle p q\rangle \leftrightarrow[q p]$ to obtain

$$
A\left(\underline{1}^{a}, 3^{-}, 4^{-}, \ldots, n^{-}, \overline{2}^{b}\right)=\frac{(-1)^{n} i m\left[\underline{1}^{a} \overline{2}^{b}\right]\left\langle 3\left|\prod_{j=3}^{n-2}\left\{\not p_{13 \ldots j} \not p_{j+1}+\left(s_{13 \ldots j}-m^{2}\right)\right\}\right| n\right\rangle}{\left(s_{13}-m^{2}\right)\left(s_{134}-m^{2}\right) \ldots\left(s_{13 \ldots(n-1)}-m^{2}\right)[34][45] \ldots[n-1 \mid n]} .
$$

\subsection{One-minus amplitudes with two quarks}

In this section we again use the on-shell recursion to derive an all-multiplicity expression

$$
\begin{aligned}
A\left(\underline{1}^{a}, 3^{-}, 4^{+}, \ldots, n^{+}, \overline{2}^{b}\right)=-\frac{i\langle 3|1| 2 \mid 3\rangle\left(\left\langle\underline{1}^{a} 3\right\rangle\left[\overline{2}^{b}|1+2| 3\right\rangle-\left\langle\overline{2}^{b} 3\right\rangle\left[\underline{1}^{a}|1+2| 3\right\rangle\right)}{s_{12}\langle 34\rangle \ldots\langle n-1 \mid n\rangle\langle 3|1| 2 \mid n\rangle} \\
\quad-\sum_{k=4}^{n-1} \frac{i m\left\langle 3\left|\not p_{1} \not \not_{3 \ldots k}\right| 3\right\rangle\left(\left\langle\underline{1}^{a} \overline{2}^{b}\right\rangle\left\langle 3\left|\not \not_{1} \not \not_{3 \ldots k}\right| 3\right\rangle+\left\langle\underline{1}^{a} 3\right\rangle\left\langle\overline{2}^{b} 3\right\rangle s_{3 \ldots k}\right)}{s_{3 \ldots k}\left(s_{13 \ldots k}-m^{2}\right) \ldots\left(s_{13 \ldots(n-1)}-m^{2}\right)\langle 34\rangle \ldots\langle k-1 \mid k\rangle\left\langle 3\left|\not \not_{1} \not p_{3 \ldots k}\right| k\right\rangle} \\
\quad \times \frac{\left\langle 3\left|\not \not_{3 \ldots k} \prod_{j=k}^{n-2}\left\{\not \not_{13 \ldots j} \not p_{j+1}+\left(s_{13 \ldots j}-m^{2}\right)\right\}\right| n\right]}{\left\langle 3\left|\not p_{1} \not \not_{3 \ldots k}\right| k+1\right\rangle\langle k+1 \mid k+2\rangle \ldots\langle n-1 \mid n\rangle}
\end{aligned}
$$

for the amplitude with two quarks and $n-2$ gluons. Here we assume negative helicity of the gluon 3 color-adjacent to the quark 1 , with all other gluon helicities positive, while the quark helicities are still left arbitrary. In principle, this color-ordered amplitude is enough to reconstruct the full color-dressed one-minus amplitude via the BCJ relations [18, 20], such as the four-point one in eq. (3.5). Indeed, the BCJ relations allow to fix the position of any gluon to be color-adjacent to the quark, with permutations acting on the remaining gluons, hence one may choose to fix the position of the minus-helicity gluon. Moreover, oneplus amplitudes can also be retrieved from eq. (4.8) via the conjugation rule $\langle p q\rangle \leftrightarrow[q p]$.

To prove the above formula, we use the same "[34)" shift as in eq. (3.8), which gives only two non-vanishing contributions at each step of the recursion,

$$
\begin{aligned}
A\left(\underline{1}^{a}, 3^{-}, 4^{+}, \ldots, n^{+}, \overline{2}^{b}\right)= & A\left(\underline{1}^{a}, \hat{3}^{-},-\hat{P}_{c}\right) \frac{1}{s_{13}-m^{2}} A\left(\hat{P}^{c}, \hat{4}^{+}, 5^{+}, \ldots, \hat{n}^{+}, \overline{2}^{b}\right) \\
& +A\left(\underline{1}^{a}, \hat{3}^{-}, \hat{P}^{+}, 6^{+}, \ldots, n^{+}, \overline{2}^{b}\right) \frac{i}{s_{45}} A\left(-\hat{P}^{-}, \hat{4}^{+}, \hat{5}^{+}\right) .
\end{aligned}
$$




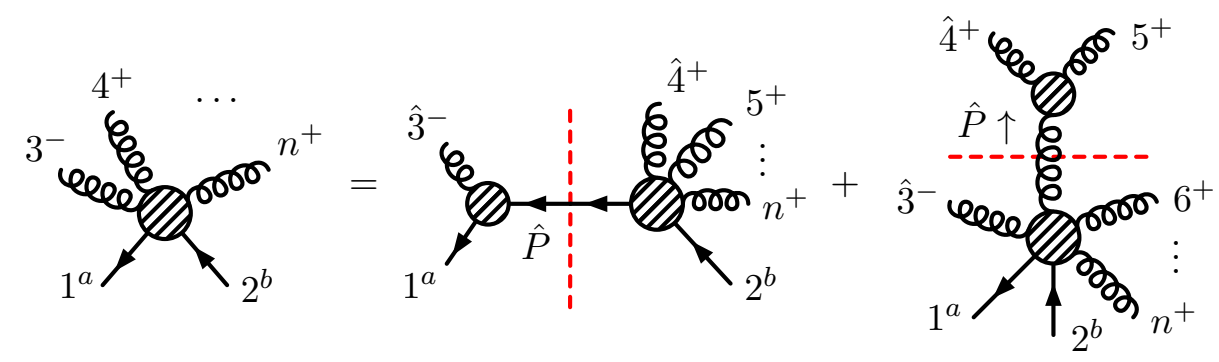

Figure 3. Graphic representation of the BCFW recursion step (4.9) for $A\left(\underline{1}^{a}, 3^{-}, 4^{+}, \ldots, n^{+}, \overline{2}^{b}\right)$.

The two residues are evaluated on the following pole kinematics:

$$
\begin{array}{llll}
z_{13}=\frac{\langle 3|1| 3]}{\langle 3|1| 4]}: & \mid \hat{3}]=-\frac{|1| 3\rangle[34]}{\langle 3|1| 4]}, \quad|\hat{4}\rangle=\frac{|3+4| 1|3\rangle}{\langle 3|1| 4]}, \quad \hat{P}^{\mu}=p_{1}^{\mu}-\frac{\left\langle 3\left|\sigma^{\mu}\right| 1|3| 4\right]}{2\langle 3|1| 4]} \\
z_{45}=-\frac{\langle 45\rangle}{\langle 35\rangle}: & \left.\mid \hat{3}]=\frac{|3+4| 5\rangle}{\langle 35\rangle}, \quad|\hat{4}\rangle=|5\rangle \frac{\langle 34\rangle}{\langle 35\rangle}, \quad|\hat{P}\rangle=|5\rangle, \quad \mid \hat{P}\right]=\frac{|4+5| 3\rangle}{\langle 53\rangle} .
\end{array}
$$

The residue at $z_{13}$ is computed immediately for any $n$ using the all-plus expresion (4.1),

$$
\begin{aligned}
A\left(\underline{1}^{a}, \hat{3}^{-},-\hat{P}^{c}\right) \frac{1}{s_{13}-m^{2}} A\left(\hat{P}_{c}, \hat{4}^{+}, 5^{+}, \ldots, \hat{n}^{+}, \overline{2}^{b}\right) \\
\quad=\frac{\left[\underline{1}^{a} \mid-\hat{P}^{c}\right]\left\langle\hat{P}_{c} \overline{2}^{b}\right\rangle\langle 3|1| q]\left[4\left|\prod_{j=4}^{n-2}\left\{\hat{p}_{P 4 \ldots j} \not p_{j+1}+\left(\hat{s}_{P 4 \ldots j}-m^{2}\right)\right\}\right| n\right]}{\left(s_{13}-m^{2}\right)\left(\hat{s}_{P 4}-m^{2}\right) \ldots\left(\hat{s}_{P 4 \ldots(n-1)}-m^{2}\right)[\hat{3} q]\langle\hat{4} 5\rangle\langle 56\rangle \ldots\langle n-1 \mid n\rangle} \\
\quad=\frac{i m\langle 3|1| 4]\left[4\left|\prod_{j=4}^{n-2}\left\{\not p_{13 \ldots j} \not p_{j+1}+\left(s_{13 \ldots j}-m^{2}\right)\right\}\right| n\right]\left(\left\langle\underline{1}^{a} \overline{2}^{b}\right\rangle\langle 3|1| 4]+\left\langle\underline{1}^{a} 3\right\rangle\left\langle\overline{2}^{b} 3\right\rangle[34]\right)}{\left(s_{13}-m^{2}\right)\left(s_{134}-m^{2}\right) \ldots\left(s_{13 \ldots(n-1)}-m^{2}\right)[34]\langle 3|1| 3+4 \mid 5\rangle\langle 56\rangle \ldots\langle n-1 \mid n\rangle} .
\end{aligned}
$$

Here the $q$-dependent factors explicitly canceled after using eq. (4.10a), and we also simplified

$$
\left[\underline{1}^{a} \mid-\hat{P}^{c}\right]\left\langle\hat{P}_{c} \overline{2}^{b}\right\rangle=-i\left[\underline{1}^{a}|\hat{P}| \overline{2}^{b}\right\rangle=\frac{i m}{\langle 3|1| 4]}\left(\left\langle\underline{1}^{a} \overline{2}^{b}\right\rangle\langle 3|1| 4]+\left\langle\underline{1}^{a} 3\right\rangle\left\langle\overline{2}^{b} 3\right\rangle[34]\right) .
$$

The contribution (4.11) from $z_{13}$ in fact coincides with the $k=4$ term of the $\sum_{k=4}^{n-1}$ sum in the full formula (4.8). To see that, we only need to rewrite

$$
\begin{gathered}
\frac{\left\langle 3\left|\not p_{1} \not p_{34}\right| 3\right\rangle}{s_{34}\langle 34\rangle\left\langle 3\left|\not p_{1} \not p_{34}\right| 4\right\rangle}\left(\left\langle\underline{1}^{a} \overline{2}^{b}\right\rangle\left\langle 3\left|\not p_{1} \not b_{34}\right| 3\right\rangle+\left\langle\underline{1}^{a} 3\right\rangle\left\langle\overline{2}^{b} 3\right\rangle s_{34}\right)\langle 3| \not p_{34} \\
=-\frac{\langle 3|1| 4]}{\left(s_{13}-m^{2}\right)[34]}\left(\left\langle\underline{1}^{a} \overline{2}^{b}\right\rangle\langle 3|1| 4]+\left\langle\underline{1}^{a} 3\right\rangle\left\langle\overline{2}^{b} 3\right\rangle[34]\right)[4 \mid .
\end{gathered}
$$

Now we turn to the residue $(4.9 \mathrm{~b})$ at $z_{45}$. First of all, we observe that the right-hand three-gluon amplitude is invariably

$$
\frac{i}{s_{45}} A\left(-\hat{P}^{-}, \hat{4}^{+}, \hat{5}^{+}\right)=\frac{i}{s_{45}} \frac{-i[45]^{4}}{[-\hat{P} \mid 4][45][5 \mid-\hat{P}]}=-\frac{[45]^{2}}{\langle 45\rangle[4 \hat{P}][5 \hat{P}]}=\frac{\langle 35\rangle}{\langle 34\rangle\langle 45\rangle} .
$$

Furthermore, in the left-hand amplitude of eq. $(4.9 \mathrm{~b}), A\left(\underline{1}^{a}, \hat{3}^{-}, \hat{P}^{+}, 6^{+}, \ldots, n^{+}, \overline{2}^{b}\right)$, most momentum sums (and their squares) are simply shifted:

$$
p_{3 \ldots(k-1)} \rightarrow \hat{p}_{3 P 6 \ldots k}=\hat{p}_{3}+\hat{p}_{4}+p_{5}+p_{6}+\ldots+p_{k}=p_{3 \ldots k}, \quad s_{3 \ldots(k-1)} \rightarrow s_{3 \ldots k} .
$$


We can then compute the residue at $z_{45}$ as

$$
\begin{aligned}
& A\left(\underline{1}^{a}, \hat{3}^{-}, \hat{P}^{+}, 6^{+}, \ldots, n^{+}, \overline{2}^{b}\right) \frac{i}{s_{45}} A\left(-\hat{P}^{-}, \hat{4}^{+}, \hat{5}^{+}\right) \\
& =\left\{-\frac{i\langle 3|1| 2 \mid 3\rangle\left(\left\langle\underline{1}^{a} 3\right\rangle\left[\overline{2}^{b}|1+2| 3\right\rangle-\left\langle\overline{2}^{b} 3\right\rangle\left[\underline{1}^{a}|1+2| 3\right\rangle\right)}{s_{12}\langle 3 \hat{P}\rangle\langle\hat{P} 6\rangle\langle 67\rangle \ldots\langle n-1 \mid n\rangle\langle 3|1| 2 \mid n\rangle}\right. \\
& -\frac{i m\left\langle 3\left|\not p_{1} \not \not_{345}\right| 3\right\rangle\left(\left\langle\underline{1}^{a} \overline{2}^{b}\right\rangle\left\langle 3\left|\not \not_{1} \not \not_{345}\right| 3\right\rangle+\left\langle\underline{1}^{a} 3\right\rangle\left\langle\overline{2}^{b} 3\right\rangle s_{345}\right)}{s_{345}\left(s_{1345}-m^{2}\right) \ldots\left(s_{13 \ldots(n-1)}-m^{2}\right)\langle 3 \hat{P}\rangle\left\langle 3\left|\not p_{1} \not p_{345}\right| \hat{P}\right\rangle} \\
& \times \frac{\left\langle 3\left|\not p_{345}\left\{\not p_{1345} \not p_{6}+\left(s_{1345}-m^{2}\right)\right\} \prod_{j=6}^{n-2}\left\{\not \not_{13 \ldots j} \not p_{j+1}+\left(s_{13 \ldots j}-m^{2}\right)\right\}\right| n\right]}{\left\langle 3\left|\not p_{1} \not p_{345}\right| 6\right\rangle\langle 67\rangle \ldots\langle n-1 \mid n\rangle} \\
& -\sum_{k=6}^{n-1} \frac{i m\left\langle 3\left|\not p_{1} \not \not_{3 \ldots k}\right| 3\right\rangle\left(\left\langle\underline{1}^{a} \overline{2}^{b}\right\rangle\left\langle 3\left|\not \not_{1} \not \not_{3 \ldots k}\right| 3\right\rangle+\left\langle\underline{1}^{a} 3\right\rangle\left\langle\overline{2}^{b} 3\right\rangle s_{3 \ldots k}\right)}{s_{3 \ldots k}\left(s_{13 \ldots k}-m^{2}\right) \ldots\left(s_{13 \ldots(n-1)}-m^{2}\right)\langle 3 \hat{P}\rangle\langle\hat{P} 6\rangle\langle 67\rangle \ldots\langle k-1 \mid k\rangle\left\langle 3\left|\not p_{1} \not \not_{3 \ldots k}\right| k\right\rangle} \\
& \left.\times \frac{\left.\left\langle 3\left|\not \not_{3 \ldots k}\right| \prod_{j=k}^{n-2}\left\{\not \not_{13 \ldots j} \not \not_{j+1}+\left(s_{13 \ldots j}-m^{2}\right)\right\}\right| n\right]}{\left\langle 3\left|\not p_{1} \not p_{3 \ldots k}\right| k+1\right\rangle\langle k+1 \mid k+2\rangle \ldots\langle n-1 \mid n\rangle}\right\} \frac{\langle 35\rangle}{\langle 34\rangle\langle 45\rangle} \\
& =-\frac{i\langle 3|1| 2 \mid 3\rangle\left(\left\langle\underline{1}^{a} 3\right\rangle\left[\overline{2}^{b}|1+2| 3\right\rangle-\left\langle\overline{2}^{b} 3\right\rangle\left[\underline{1}^{a}|1+2| 3\right\rangle\right)}{s_{12}\langle 34\rangle\langle 45\rangle\langle 56\rangle\langle 67\rangle \ldots\langle n-1 \mid n\rangle\langle 3|1| 2 \mid n\rangle} \\
& -\sum_{k=5}^{n-1} \frac{i m\left\langle 3\left|\not p_{1} \not p_{3 \ldots k}\right| 3\right\rangle\left(\left\langle\underline{1}^{a} \overline{2}^{b}\right\rangle\left\langle 3\left|\not p_{1} \not p_{3 \ldots k}\right| 3\right\rangle+\left\langle\underline{1}^{a} 3\right\rangle\left\langle\overline{2}^{b} 3\right\rangle s_{3 \ldots k}\right)}{s_{3 \ldots k}\left(s_{13 \ldots k}-m^{2}\right) \ldots\left(s_{13 \ldots(n-1)}-m^{2}\right)\langle 34\rangle\langle 45\rangle\langle 56\rangle\langle 67\rangle \ldots\langle k-1 \mid k\rangle\left\langle 3\left|\not p_{1} \not p_{3 \ldots k}\right| k\right\rangle} \\
& \times \frac{\left\langle 3\left|\not p_{3 \ldots k} \prod_{j=k}^{n-2}\left\{\not p_{13 \ldots j} \not \not_{j+1}+\left(s_{13 \ldots j}-m^{2}\right)\right\}\right| n\right]}{\left\langle 3\left|\not p_{1} \not p_{3 \ldots k}\right| k+1\right\rangle\langle k+1 \mid k+2\rangle \ldots\langle n-1 \mid n\rangle} \text {. }
\end{aligned}
$$

Here we were able to integrate the second term in the bracket (4.16a), which corresponds to $k=\hat{P}$, into the $\sum_{k=6}^{n-1}$ sum as that for $k=5$. Since the $k=4$ term, missing from eq. (4.16), is provided by the residue at $z_{13}$, this concludes the proof of the formula (4.8).

\section{Checks}

As the first simple check of our all-multiplicity formulae (4.1) and (4.8), we evaluate their massless limits. The former explicitly vanishes, as it should, whereas the latter reduces to the massless MHV amplitudes with two quarks:

$$
A\left(\underline{1}^{a}, 3^{-}, 4^{+}, \ldots, n^{+}, \overline{2}^{b}\right) \underset{m \rightarrow 0}{\longrightarrow} \frac{-i}{\langle 13\rangle\langle 34\rangle\langle 45\rangle \ldots\langle n-1 \mid n\rangle\langle n \mid 2\rangle\langle 21\rangle}\left(\begin{array}{cc}
0 & \langle 13\rangle\langle 23\rangle^{3} \\
\langle 13\rangle^{3}\langle 23\rangle & 0
\end{array}\right) .
$$

This analytic check, however, is only sensitive to a single term in eq. (4.8) that is not multiplied by the mass. As another partial check, we happened to have a six-point Feynmandiagrammatic calculation at easy access, with which we found numerical agreement to ten significant digits for both helicity configurations. Needless to say, the Feynman diagrams were much lengthier before evaluation than the three-term amplitude generated by the formula (4.8). The all-plus formula (4.1) can also be independently verified via the 
fundamental BCJ relation [18, 20, 21, 27]

$$
\sum_{i=2}^{n-1}\left(p_{13 \ldots i} \cdot p_{n}\right) A\left(\underline{1}^{a}, 3^{+}, \ldots, i^{+}, n^{+},(i+1)^{+}, \ldots,(n-1)^{+}, \overline{2}^{b}\right)=0
$$

which is non-trivially satisfied by a linear combination of its permutations. We checked it numerically to ten significant digits for $n=4,5, \ldots, 12$. Let us now turn to even more stringent checks.

In ref. [14] Schwinn and Weinzierl used a massive extension [8-10] of the massless spinor-helicity formalism to compute QCD amplitudes with the same gluon polarizations that we compute here. In that formalism, the massive spinors are introduced by expanding the massive momentum $p_{\mu}$ in terms of its massless "flat" version $p_{\mu}^{b}$ and another null vector $q_{\mu}$ :

$$
p_{\mu}=p_{\mu}^{b}+\frac{m^{2}}{2(p \cdot q)} q_{\mu}, \quad u_{p}^{+}(q)=\frac{(\not p+m)|q\rangle}{\left\langle p^{b} q\right\rangle}, \quad u_{p}^{-}(q)=\frac{(\not p+m) \mid q]}{\left[p^{b} q\right]} .
$$

The reference momentum $q_{\mu}$ determines the spin quantization axis, since the resulting spin vector is $\vec{s}^{ \pm}= \pm\left\{\hat{p} / 2-m^{2} \vec{q} / 4|\vec{p}| p \cdot q\right\}$. This shows that unless $m \neq 0$ the quark labels \pm in the spinors (5.3) are not helicities but rather general spin labels.

The versatility of the massive spinor-helicity formalism [11] reviewed in section 2 lets us effortlessly pick any quantization axis in the sense of eq. (5.3). We simply need to switch from the helicity parametrization (2.16) to

$$
\begin{aligned}
& u_{p}^{a=1}=\left(\begin{array}{l}
\left|p^{1}\right\rangle \equiv \frac{m|q\rangle}{\left\langle p^{b} q\right\rangle} \\
\left.\left.\mid p^{1}\right] \equiv \mid p^{b}\right]
\end{array}\right)=u_{p}^{+}(q), \quad \bar{u}_{p, a=1}=\left(\begin{array}{c}
\left\langle p^{2}\right| \equiv\left\langle p^{b}\right| \\
-\left[p^{2} \mid \equiv \frac{m[q \mid}{\left[q p^{b}\right]}\right.
\end{array}\right)=\bar{u}_{p}^{+}(q), \\
& u_{p}^{a=2}=\left(\begin{array}{c}
\left|p^{2}\right\rangle \equiv\left|p^{b}\right\rangle \\
\left.\mid p^{2}\right] \equiv \frac{m \mid q]}{\left[p^{p} q\right]}
\end{array}\right)=u_{p}^{-}(q), \quad \bar{u}_{p, a=2}=\left(\begin{array}{c}
-\left\langle p^{1}\right| \equiv \frac{m\langle q|}{\left\langle q p^{\dagger}\right\rangle} \\
{\left[p^{1} \mid \equiv\left[p^{b} \mid\right.\right.}
\end{array}\right)=\bar{u}_{p}^{-}(q) .
\end{aligned}
$$

Now if we take the same reference vector $q^{\mu}$ for both quarks, this dictionary gives us

$$
\left\langle\underline{1}^{a} \overline{2}^{b}\right\rangle=\left(\begin{array}{cc}
0 & -m \frac{\left\langle q 2^{b}\right\rangle}{\left\langle q 1^{b}\right\rangle} \\
-m \frac{\left\langle 1^{b} q\right\rangle}{\left\langle 2^{b} q\right\rangle} & \left\langle 1^{b} 2^{b}\right\rangle
\end{array}\right), \quad\left\langle\underline{1}^{a} 3\right\rangle\left\langle\overline{2}^{b} 3\right\rangle=\left(\begin{array}{cc}
0 & -\frac{m\langle q 3\rangle}{\left\langle q 1^{b}\right\rangle}\left\langle 2^{b} 3\right\rangle \\
\left\langle 1^{b} 3\right\rangle \frac{m\langle q 3\rangle}{\left\langle q 2^{b}\right\rangle} & \left\langle 1^{b} 3\right\rangle\left\langle 2^{b} 3\right\rangle
\end{array}\right),
$$

where the signs follow from our convention $m_{2}=-m_{1}=-m$.

Ref. [14] actually sets $q^{\mu}$ to the momentum of the minus-helicity gluon 3 . This allowed for BCFW shifts involving this pair of massive and massless momenta, and thus set up a recursion to compute the amplitudes in question. Let us translate the results of ref. [14] 
to the conventions of the present paper:

$$
\begin{aligned}
& A\left(\underline{1}^{1}, 3^{-}, 4^{+}, \ldots, n^{+}, \overline{2}^{1}\right)=0, \\
& A\left(\underline{1}^{1}, 3^{-}, 4^{+}, \ldots, n^{+}, \overline{2}^{2}\right)=\frac{i\left\langle 2^{b} 3\right\rangle}{\left\langle 1^{b} 3\right\rangle\langle 34\rangle \ldots\langle n-1 \mid n\rangle} \sum_{k=4}^{n} \frac{\left\langle 3\left|\not p_{1} \not p_{3 \ldots k}\right| 3\right\rangle^{2}}{s_{3 \ldots k}\left\langle 3\left|\not p_{1} \not p_{3 \ldots k}\right| k\right\rangle} \\
& \times\left\{\delta_{k=n}+\delta_{k \neq n} \frac{m^{2}\langle k \mid k+1\rangle\left\langle 3\left|\not p_{3 \ldots k} \prod_{j=k+1}^{n-1}\left\{\left(s_{13 \ldots j}-m^{2}\right)-\not p_{j} \not p_{13 \ldots j}\right\}\right| n\right]}{\left(s_{13 \ldots k}-m^{2}\right) \ldots\left(s_{13 \ldots(n-1)}-m^{2}\right)\left\langle 3\left|\not \not_{1} \not p_{3 \ldots k}\right| k+1\right\rangle}\right\}, \\
& A\left(\underline{1}^{2}, 3^{-}, 4^{+}, \ldots, n^{+}, \overline{2}^{1}\right)=\frac{i\left\langle 1^{b} 3\right\rangle}{\left\langle 2^{b} 3\right\rangle\langle 34\rangle \ldots\langle n-1 \mid n\rangle} \sum_{k=4}^{n} \frac{\left\langle 3\left|\not p_{1} \not p_{3 \ldots k}\right| 3\right\rangle^{2}}{s_{3 \ldots k}\left\langle 3\left|\not p_{1} \not p_{3 \ldots k}\right| k\right\rangle} \\
& \times\left\{\delta_{k=n}+\delta_{k \neq n} \frac{m^{2}\langle k \mid k+1\rangle\left\langle 3\left|\not p_{3 \ldots k} \prod_{j=k+1}^{n-1}\left\{\left(s_{13 \ldots j}-m^{2}\right)-\not p_{j} \not p_{13 \ldots j}\right\}\right| n\right]}{\left(s_{13 \ldots k}-m^{2}\right) \ldots\left(s_{13 \ldots(n-1)}-m^{2}\right)\left\langle 3\left|\not \not_{1} \not p_{3 \ldots k}\right| k+1\right\rangle}\right\}, \\
& A\left(\underline{1}^{2}, 3^{-}, 4^{+}, \ldots, n^{+}, \overline{2}^{2}\right)=\frac{-i\left\langle 1^{b} 2^{b}\right\rangle}{m\langle 34\rangle \ldots\langle n-1 \mid n\rangle} \sum_{k=4}^{n} \frac{\left\langle 3\left|\not p_{1} \not p_{3 \ldots k}\right| 3\right\rangle^{2}}{s_{3 \ldots k}\left\langle 3\left|\not p_{1} \not p_{3 \ldots k}\right| k\right\rangle}\left[1+\frac{s_{3 \ldots k}\left\langle 32^{b}\right\rangle}{\left\langle 3\left|\not p_{3 \ldots k} \not p_{1}^{b}\right| 2^{b}\right\rangle}\right] \\
& \times\left\{\delta_{k=n}+\delta_{k \neq n} \frac{m^{2}\langle k \mid k+1\rangle\left\langle 3\left|\not \not_{3 \ldots k} \prod_{j=k+1}^{n-1}\left\{\left(s_{13 \ldots j}-m^{2}\right)-\not p_{j} \not \not_{13 \ldots j}\right\}\right| n\right]}{\left(s_{13 \ldots k}-m^{2}\right) \ldots\left(s_{13 \ldots(n-1)}-m^{2}\right)\left\langle 3\left|\not \not_{1} \not p_{3 \ldots k}\right| k+1\right\rangle}\right\} .
\end{aligned}
$$

To make a direct comparison easier, here we rewrite our result (4.8) as

$$
\begin{gathered}
A\left(\underline{1}^{a}, 3^{-}, 4^{+}, \ldots, n^{+}, \overline{2}^{b}\right) \\
=\frac{-i}{\langle 34\rangle \ldots\langle n-1 \mid n\rangle}\left\{\frac{\left\langle 3\left|\not p_{1} \not \not_{3 \ldots n}\right| 3\right\rangle}{m s_{3 \ldots n}\left\langle 3\left|\not p_{1} \not p_{3 \ldots k}\right| n\right\rangle}\left(\left\langle\underline{1}^{a} \overline{2}^{b}\right\rangle\left\langle 3\left|\not p_{1} \not p_{3 \ldots n}\right| 3\right\rangle+\left\langle\underline{1}^{a} 3\right\rangle\left\langle\overline{2}^{b} 3\right\rangle s_{3 \ldots n}\right)\right. \\
+m \sum_{k=4}^{n-1} \frac{\langle k \mid k+1\rangle\left\langle 3\left|\not p_{1} \not p_{3 \ldots k}\right| 3\right\rangle\left\langle 3\left|\not p_{3 \ldots k} \prod_{j=k}^{n-2}\left\{\left(s_{13 \ldots(j+1)}-m^{2}\right)-\not p_{j+1} \not p_{13 \ldots(j+1)}\right\}\right| n\right]}{s_{3 \ldots k}\left(s_{13 \ldots k}-m^{2}\right) \ldots\left(s_{13 \ldots(n-1)}-m^{2}\right)\left\langle 3\left|\not p_{1} \not p_{3 \ldots k}\right| k\right\rangle\left\langle 3\left|\not p_{1} \not p_{3 \ldots k}\right| k+1\right\rangle} \\
\left.\times\left(\left\langle\underline{1}^{a} \overline{2}^{b}\right\rangle\left\langle 3\left|\not p_{1} \not \not_{3 \ldots k}\right| 3\right\rangle+\left\langle\underline{1}^{a} 3\right\rangle\left\langle\overline{2}^{b} 3\right\rangle s_{3 \ldots k}\right)\right\},
\end{gathered}
$$

where we massaged the product $\prod_{j=k}^{n-2}$ into the form of eq. (5.6) using the anticommutator identity

$$
\not p_{13 \ldots j} \not \not_{j+1}+\left(s_{13 \ldots j}-m^{2}\right)=\left(s_{13 \ldots(j+1)}-m^{2}\right)-\not p_{j+1} \not \not_{13 \ldots j} \text {. }
$$

Now we can see with a naked eye that our formula (5.7) exactly reproduces eqs. (5.6a), (5.6b) and (5.6c), where all the terms containing $\left\langle\underline{1}^{a} 3\right\rangle\left\langle\overline{2}^{b} 3\right\rangle$ vanish due to the choice $q=p_{3}$ for both quarks. To match the last amplitude (5.6d), for which $\left|\underline{1}^{a=2}\right\rangle=\left|1^{b}\right\rangle$, $\left|\overline{2}^{b=2}\right\rangle=\left|2^{b}\right\rangle$, we observe that

$$
\left\langle 1^{b} 2^{b}\right\rangle\left\langle 3\left|\not \not_{1} \not p_{3 \ldots k}\right| 3\right\rangle+\left\langle 1^{b} 3\right\rangle\left\langle 2^{b} 3\right\rangle s_{3 \ldots k}=\left\langle 1^{b} 2^{b}\right\rangle\left\langle 3\left|\not p_{1} \not \not_{3 \ldots k}\right| 3\right\rangle\left[1+\frac{s_{3 \ldots k}\left\langle 32^{b}\right\rangle}{\left\langle 3\left|\not \not_{3 \ldots k} \not p_{1}^{b}\right| 2^{b}\right\rangle}\right] .
$$

To conclude, we note that ref. [14] also computed the analogue of the all-plus amplitude in the massless spinor-helicity formalism (5.3) through its relation to the massive scalar amplitude of refs. [28, 29] via a supersymmetric Ward identity [30-32] with an unfixed reference vector $q^{\mu}$. The same eqs. (5.5) and (5.8) allow to easily verify that these results are incorporated in our formula (4.1). 


\section{Summary and discussion}

In this note we have computed two infinite families of tree-level amplitudes with two quarks of arbitrary spin and any number of gluons with specified helicities. For that we have used the new massive spinor-helicity formalism of ref. [11]. In order to check the consistency of our results with the literature, we have also established straightforward transition rules between our approach and the more traditional ones.

We hope to have demonstrated that the new massive formalism is a analytic tool well-suited for QCD computations. It is a logical extension of the massless spinor-helicity formalism [1-6, 11], which in the last decades has become indispensable for scatteringamplitude calculations. Of course, the scope of the formalism is much more general than QCD, as shown by the recent applications to gravitational scattering [33, 34] and the Standard Model as a whole $[11,35]$. It can be used streamline the consideration of all unitarity-compliant three-point [35-37] and four-point [11] interactions. It can also be related to much earlier off-shell reformulations of QED [38, 39] and other theories [40-42] using two-component spinor fields.

The presented formalism has potential to facilitate many QCD calculations, both analytically and numerically. Through its analytic simplicity, it may provide a way to explicit expressions for tree amplitudes with more general gluon helicity configurations [43-46] and more quark-antiquark lines [17, 18], as already achieved [47, 48] for the massless QCD amplitudes with up to three quark-antiquark pairs. For example, it would be interesting to find an analytic expression even for an amplitude with two quarks and one negativehelicity gluon in an arbitrary position, provided that it is more compact than its BCJ relation $[18,20]$ that involves various permutations of the formula computed in this note.

New loop amplitudes could also be calculated using the presented formalism. Indeed, loops can be obtained from generalized unitarity cuts [49-55] that are constructed from tree amplitudes. ${ }^{7}$ It would also be interesting to investigate, in the spirit of refs. [58, 59], if the massive on-shell formalism could speed up numerical evaluation of tree-level amplitudes. This would be beneficial for computing real-emission radiative QCD corrections to a vast array of elementary-particle scattering processes.

\section{Acknowledgments}

The author would like to thank Nikolay Gulitskiy and Achilleas Lazopoulos for their valuable comments on the manuscript. This project has received funding from the European Union's Horizon 2020 research and innovation programme under the Marie SkłodowskaCurie grant agreement 746138 .

\footnotetext{
${ }^{7} \mathrm{~A}$ relevant example is the recent calculation [56] of one-loop amplitudes with external massive fermions via tree amplitudes in six dimensions. One extra dimension was needed to parametrize the loop dependence in dimensional regularization, and another one to account for the quark mass. Since the massive fourdimensional spinor helicity can be viewed as massless but five-dimensional, the amplitudes considered in this note could be embedded into a higher-dimensional formalism, such as the six-dimensional one of ref. [57].
} 


\section{A Massless spinor parametrizations}

For this note to be more self-contained, we give a realization of the massless spinors [1-6]. In terms of the light-cone momentum components

$$
p_{+}=p^{0}+p^{3}, \quad p_{-}=p^{0}-p^{3}, \quad p_{\perp}=p^{1}+i p^{2}, \quad \bar{p}_{\perp}=p^{1}-i p^{2},
$$

satisfying $p_{+} p_{-}=p_{\perp} \bar{p}_{\perp}$, an explicit solution for the Weyl spinors is (adapted from ref. [60])

$$
\lambda_{p \alpha}=\left(\begin{array}{c}
-\bar{p}_{\perp} / \sqrt{p_{+}} \\
\sqrt{p_{+}}
\end{array}\right), \quad \tilde{\lambda}_{p \dot{\alpha}}=\left(\begin{array}{c}
-p_{\perp} / \sqrt{p_{+}} \\
\sqrt{p_{+}}
\end{array}\right),
$$

which is a rewrite of eq. (2.11). If $p_{+}$happens to vanish, an equivalent solution may be used:

$$
\lambda_{p \alpha}=\left(\begin{array}{c}
-\sqrt{p_{-}} \\
p_{\perp} / \sqrt{p_{-}}
\end{array}\right), \quad \tilde{\lambda}_{p \dot{\alpha}}=\left(\begin{array}{c}
-\sqrt{p_{-}} \\
\bar{p}_{\perp} / \sqrt{p_{-}}
\end{array}\right) .
$$

In the complex-valued case where $p_{ \pm}=0$ and the momentum equals $\left(0, p^{1}, \pm i p^{1}, 0\right)$, a valid choice is

$$
\lambda_{p \alpha}=\frac{1}{\sqrt{2 p^{1}}}\left(\begin{array}{c}
-\bar{p}_{\perp} \\
p_{\perp}
\end{array}\right), \quad \tilde{\lambda}_{p \dot{\alpha}}=\frac{1}{\sqrt{2 p^{1}}}\left(\begin{array}{c}
-p_{\perp} \\
\bar{p}_{\perp}
\end{array}\right) .
$$

Note that these definitions give $\left(\lambda_{p \alpha}\right)^{*}=\tilde{\lambda}_{p \dot{\alpha}}$ for a real-valued momentum $p^{\mu}$. Moreover, for the principal square roots they are also consistent with the momentum inversion rule

$$
\lambda_{-p}=\operatorname{sgn}\left(p^{0}\right) i \lambda_{p}, \quad \tilde{\lambda}_{-p}=\operatorname{sgn}\left(p^{0}\right) i \tilde{\lambda}_{p} .
$$

\section{B Massive spinor parametrizations}

Here we give the massive spinor-helicity variables that are consistent with the parametrizations (A.2) through (A.4) in the massless limit. The spinors (2.20) can be rewritten as

$$
\begin{aligned}
& \lambda_{p \alpha}^{a}=\sqrt{\frac{E+P}{2 P}}\left(\begin{array}{c}
-\bar{p}_{\perp} / \sqrt{p_{+}} \\
\sqrt{p_{+}}
\end{array}\right)_{\alpha} \otimes\left(\begin{array}{l}
0 \\
1
\end{array}\right)^{a}+\sqrt{\frac{E-P}{2 P}}\left(\begin{array}{c}
\sqrt{p_{+}} \\
p_{\perp} / \sqrt{p_{+}}
\end{array}\right)_{\alpha} \otimes\left(\begin{array}{l}
1 \\
0
\end{array}\right)^{a}, \\
& \tilde{\lambda}_{p \dot{\alpha}}^{a}=\sqrt{\frac{E+P}{2 P}}\left(\begin{array}{c}
-p_{\perp} / \sqrt{p_{+}} \\
\sqrt{p_{+}}
\end{array}\right)_{\dot{\alpha}} \otimes\left(\begin{array}{l}
1 \\
0
\end{array}\right)^{a}-\sqrt{\frac{E-P}{2 P}}\left(\begin{array}{c}
\sqrt{p_{+}} \\
\bar{p}_{\perp} / \sqrt{p_{+}}
\end{array}\right)_{\dot{\alpha}} \otimes\left(\begin{array}{l}
0 \\
1
\end{array}\right)^{a},
\end{aligned}
$$

where now we take $p_{ \pm}=P \pm p^{3}, p_{\perp}=p^{1}+i p^{2}$. Moreover, as mentioned in section 2, we introduce sign functions in the definitions

$$
P=\operatorname{sgn}(E) \sqrt{\vec{p}^{2}}, \quad m=\operatorname{sgn}(E) \sqrt{E^{2}-P^{2}} .
$$

This allows the massless limit to keep $(E+P)$ finite and send $(E-P) \rightarrow \operatorname{sgn}(E) m^{2} /(2 P)$ for all real-valued energies $E=p^{0}$, as well as preserve $\operatorname{det}\left\{\lambda_{p \alpha}{ }_{\alpha}\right\}=\operatorname{det}\left\{\tilde{\lambda}_{p \dot{\alpha}}^{a}\right\}=m$ and the 
form (2.17) of the Dirac equation for antiparticles. If $p_{+}$happens to vanish, an equivalent solution may be used: ${ }^{8}$

$$
\begin{aligned}
& \lambda_{p \alpha}^{a}=\sqrt{\frac{E+P}{2 P}}\left(\begin{array}{c}
-\sqrt{p_{-}} \\
p_{\perp} / \sqrt{p_{-}}
\end{array}\right)_{\alpha} \otimes\left(\begin{array}{l}
0 \\
1
\end{array}\right)^{a}+\sqrt{\frac{E-P}{2 P}}\left(\begin{array}{c}
\bar{p}_{\perp} / \sqrt{p_{-}} \\
\sqrt{p_{-}}
\end{array}\right)_{\alpha} \otimes\left(\begin{array}{l}
1 \\
0
\end{array}\right)^{a}, \\
& \tilde{\lambda}_{p \dot{\alpha}}^{a}=\sqrt{\frac{E+P}{2 P}}\left(\begin{array}{c}
-\sqrt{p_{-}} \\
\bar{p}_{\perp} / \sqrt{p_{-}}
\end{array}\right)_{\dot{\alpha}} \otimes\left(\begin{array}{l}
1 \\
0
\end{array}\right)^{a}-\sqrt{\frac{E-P}{2 P}}\left(\begin{array}{c}
p_{\perp} / \sqrt{p_{-}} \\
\sqrt{p_{-}}
\end{array}\right)_{\dot{\alpha}} \otimes\left(\begin{array}{l}
0 \\
1
\end{array}\right)^{a} .
\end{aligned}
$$

Another singular region is where $P=0$, and thus $p^{0}=m$ and $p_{ \pm}= \pm p^{3}$. Then we choose

$$
\begin{aligned}
& \lambda_{p \alpha}^{a}=\sqrt{\bar{p}_{\perp}}\left(\begin{array}{c}
\sqrt{\left(m-p_{+}\right) p_{\perp}} \\
-\sqrt{p_{\perp} /\left(m-p_{+}\right)}
\end{array}\right) \otimes\left(\begin{array}{l}
0 \\
1
\end{array}\right)^{a}+\frac{m}{\sqrt{m-p_{+}}}\left(\begin{array}{c}
0 \\
-\sqrt{p_{\perp} / \bar{p}_{\perp}}
\end{array}\right)_{\alpha} \otimes\left(\begin{array}{l}
1 \\
0
\end{array}\right)^{a}, \\
& \tilde{\lambda}_{p \dot{\alpha}}^{a}=\sqrt{p_{\perp}}\left(\begin{array}{c}
\sqrt{\left(m-p_{+}\right) / \bar{p}_{\perp}} \\
-\sqrt{\bar{p}_{\perp} /\left(m-p_{+}\right)}
\end{array}\right) \otimes\left(\begin{array}{l}
1 \\
0
\end{array}\right)^{a}-\frac{m}{\sqrt{m-p_{+}}}\left(\begin{array}{c}
0 \\
-\sqrt{\bar{p}_{\perp} / p_{\perp}}
\end{array}\right)_{\dot{\alpha}} \otimes\left(\begin{array}{l}
0 \\
1
\end{array}\right)^{a},
\end{aligned}
$$

the massless limit of which is consistent with that of eq. (B.1) (evaluated at $P=0$ ). These spinors do not, however, correspond to definite helicities, as the spin vector defined by eq. (2.19) turns out to be $\vec{s}_{a}=(-1)^{a-1}\left\{\vec{p} / 2 m^{-}\left(0,0, \frac{m}{2\left(m-p_{+}\right)}\right)\right\}$for a complex $p^{\mu}$. Similarly, in the case where $p_{ \pm}=0$ and the momentum equals $\left(m, p^{1}, \pm i p^{1}, 0\right)$, a valid choice is

$$
\begin{aligned}
& \lambda_{p \alpha}^{a}=\frac{1}{\sqrt{2 p^{1}}}\left(\begin{array}{c}
-\bar{p}_{\perp}+m \\
p_{\perp}-m
\end{array}\right)_{\alpha} \otimes\left(\begin{array}{l}
0 \\
1
\end{array}\right)^{a}+\frac{1}{\sqrt{2 p^{1}}}\left(\begin{array}{c}
\mp m \\
\pm m
\end{array}\right)_{\alpha} \otimes\left(\begin{array}{l}
1 \\
0
\end{array}\right)^{a}, \\
& \tilde{\lambda}_{p \dot{\alpha}}^{a}=\frac{1}{\sqrt{2 p^{1}}}\left(\begin{array}{c}
-p_{\perp} \mp m \\
\bar{p}_{\perp} \mp m
\end{array}\right)_{\dot{\alpha}} \otimes\left(\begin{array}{l}
1 \\
0
\end{array}\right)^{a}-\frac{1}{\sqrt{2 p^{1}}}\left(\begin{array}{c}
2 p_{\perp}-m \\
2 \bar{p}_{\perp}-m
\end{array}\right)_{\dot{\alpha}} \otimes\left(\begin{array}{l}
0 \\
1
\end{array}\right)^{a},
\end{aligned}
$$

yielding the spin vectors $\vec{s}_{a}=(-1)^{a-1}\left\{\vec{p} / 2 m-\left(1, \pm i\left(1-m / 2 p^{1}\right), \pm\left(1-m / 2 p^{1}\right)\right\}\right.$. For a real massive momentum at rest $p^{\mu}=(m, 0,0,0)$ we choose

$$
\lambda_{p \alpha}^{a}=\left(\begin{array}{c}
0 \\
\sqrt{m}
\end{array}\right)_{\alpha} \otimes\left(\begin{array}{l}
0 \\
1
\end{array}\right)^{a}+\left(\begin{array}{c}
\sqrt{m} \\
0
\end{array}\right)_{\alpha} \otimes\left(\begin{array}{l}
1 \\
0
\end{array}\right)^{a}, \quad \tilde{\lambda}_{p \dot{\alpha}}^{a}=\left(\begin{array}{c}
0 \\
\sqrt{m}
\end{array}\right)_{\dot{\alpha}} \otimes\left(\begin{array}{l}
1 \\
0
\end{array}\right)^{a}-\left(\begin{array}{c}
\sqrt{m} \\
0
\end{array}\right)_{\dot{\alpha}} \otimes\left(\begin{array}{l}
0 \\
1
\end{array}\right)^{a} .
$$

This choice naturally aligns the spin vector $\vec{s}^{a}=(-1)^{a-1}(0,0,1 / 2)$ with the $z$-axis. Finally, we note that for real-valued momenta $p^{\mu}$ the above definitions satisfy $\left(\lambda_{p \alpha}\right)^{*}=\tilde{\lambda}_{p \dot{\alpha}}$ and $\left(\eta_{p \alpha}\right)^{*}=\tilde{\eta}_{p \dot{\alpha}}$ and are consistent with the momentum inversion rule

$$
\lambda_{-p}=\operatorname{sgn}\left(p^{0}\right) i \lambda_{p}, \quad \tilde{\lambda}_{-p}=\operatorname{sgn}\left(p^{0}\right) i \tilde{\lambda}_{p}
$$

Open Access. This article is distributed under the terms of the Creative Commons Attribution License (CC-BY 4.0), which permits any use, distribution and reproduction in any medium, provided the original author(s) and source are credited.

\footnotetext{
${ }^{8}$ Using that $\sqrt{p_{+}}=\sqrt{2 P} \cos (\theta / 2), \sqrt{p_{-}}=\sqrt{2 P} \sin (\theta / 2)$ and $p_{\perp}=2 P e^{i \varphi} \sin (\theta / 2) \cos (\theta / 2)$, one can relate the parametrizations (B.1) and (B.3) by the spin-preserving little-group rotation $\omega_{b}^{a}=\left(\begin{array}{cc}e^{-i \varphi} & 0 \\ 0 & e^{i \varphi}\end{array}\right)$.
} 


\section{References}

[1] F.A. Berends, R. Kleiss, P. De Causmaecker, R. Gastmans and T.T. Wu, Single bremsstrahlung processes in gauge theories, Phys. Lett. B 103 (1981) 124 [InSPIRE].

[2] P. De Causmaecker, R. Gastmans, W. Troost and T.T. Wu, Multiple bremsstrahlung in gauge theories at high-energies. 1. General formalism for quantum electrodynamics, Nucl. Phys. B 206 (1982) 53 [INSPIRE].

[3] J.F. Gunion and Z. Kunszt, Improved analytic techniques for tree graph calculations and the

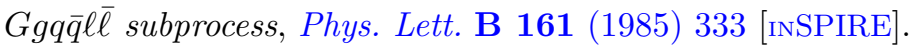

[4] R. Kleiss and W.J. Stirling, Spinor techniques for calculating $p \bar{p} \rightarrow W^{ \pm} / Z^{0}+$ jets, Nucl. Phys. B 262 (1985) 235 [InSPIRE].

[5] Z. Xu, D.-H. Zhang and L. Chang, Helicity amplitudes for multiple bremsstrahlung in massless non-Abelian gauge theories, Nucl. Phys. B 291 (1987) 392 [INSPIRE].

[6] R. Gastmans and T.T. Wu, The ubiquitous photon: helicity method for QED and QCD, Int. Ser. Monogr. Phys. 80 (1990) 1 [INSPIRE].

[7] L.J. Dixon, Calculating scattering amplitudes efficiently, in QCD and beyond. Proceedings, Theoretical Advanced Study Institute in Elementary Particle Physics, TASI-95, SLAC-PUB-7106, Boulder CO U.S.A., 4-30 June 1995, p. 539 [hep-ph/9601359] [INSPIRE].

[8] R. Kleiss and W.J. Stirling, Cross-sections for the production of an arbitrary number of photons in electron-positron annihilation, Phys. Lett. B 179 (1986) 159 [INSPIRE].

[9] S. Dittmaier, Weyl-van der Waerden formalism for helicity amplitudes of massive particles, Phys. Rev. D 59 (1998) 016007 [hep-ph/9805445] [INSPIRE].

[10] C. Schwinn and S. Weinzierl, Scalar diagrammatic rules for Born amplitudes in QCD, JHEP 05 (2005) 006 [hep-th/0503015] [INSPIRE].

[11] N. Arkani-Hamed, T.-C. Huang and Y.-T. Huang, Scattering amplitudes for all masses and spins, arXiv: 1709.04891 [INSPIRE].

[12] R. Britto, F. Cachazo and B. Feng, New recursion relations for tree amplitudes of gluons, Nucl. Phys. B 715 (2005) 499 [hep-th/0412308] [INSPIRE].

[13] R. Britto, F. Cachazo, B. Feng and E. Witten, Direct proof of tree-level recursion relation in Yang-Mills theory, Phys. Rev. Lett. 94 (2005) 181602 [hep-th/0501052] [INSPIRE].

[14] C. Schwinn and S. Weinzierl, On-shell recursion relations for all Born QCD amplitudes, JHEP 04 (2007) 072 [hep-ph/0703021] [INSPIRE].

[15] E.P. Wigner, On unitary representations of the inhomogeneous Lorentz group, Annals Math. 40 (1939) 149.

[16] V. Bargmann and E.P. Wigner, Group theoretical discussion of relativistic wave equations, Proc. Nat. Acad. Sci. 34 (1948) 211 [inSPIRE].

[17] H. Johansson and A. Ochirov, Pure gravities via color-kinematics duality for fundamental matter, JHEP 11 (2015) 046 [arXiv: 1407.4772] [INSPIRE].

[18] H. Johansson and A. Ochirov, Color-kinematics duality for QCD amplitudes, JHEP 01 (2016) 170 [arXiv:1507.00332] [INSPIRE].

[19] R. Kleiss and H. Kuijf, Multi-gluon cross-sections and five jet production at hadron colliders, Nucl. Phys. B 312 (1989) 616 [inSPIRE].

[20] Z. Bern, J.J.M. Carrasco and H. Johansson, New relations for gauge-theory amplitudes, Phys. Rev. D 78 (2008) 085011 [arXiv:0805.3993] [INSPIRE]. 
[21] L. de la Cruz, A. Kniss and S. Weinzierl, Proof of the fundamental BCJ relations for QCD amplitudes, JHEP 09 (2015) 197 [arXiv:1508.01432] [INSPIRE].

[22] V. Del Duca, L.J. Dixon and F. Maltoni, New color decompositions for gauge amplitudes at tree and loop level, Nucl. Phys. B 571 (2000) 51 [hep-ph/9910563] [inSPIRE].

[23] N.E.J. Bjerrum-Bohr, J.F. Donoghue and P. Vanhove, On-shell techniques and universal results in quantum gravity, JHEP 02 (2014) 111 [arXiv:1309.0804] [INSPIRE].

[24] S.J. Parke and T.R. Taylor, An amplitude for $n$ gluon scattering, Phys. Rev. Lett. 56 (1986) 2459 [INSPIRE].

[25] N. Arkani-Hamed and J. Kaplan, On tree amplitudes in gauge theory and gravity, JHEP 04 (2008) 076 [arXiv:0801.2385] [InSPIRE].

[26] R. Britto and A. Ochirov, On-shell recursion for massive fermion currents, JHEP 01 (2013) 002 [arXiv: 1210.1755] [INSPIRE].

[27] B. Feng, R. Huang and Y. Jia, Gauge amplitude identities by on-shell recursion relation in S-matrix program, Phys. Lett. B 695 (2011) 350 [arXiv: 1004.3417] [INSPIRE].

[28] D. Forde and D.A. Kosower, All-multiplicity amplitudes with massive scalars, Phys. Rev. D 73 (2006) 065007 [hep-th/0507292] [INSPIRE].

[29] P. Ferrario, G. Rodrigo and P. Talavera, Compact multigluonic scattering amplitudes with heavy scalars and fermions, Phys. Rev. Lett. 96 (2006) 182001 [hep-th/0602043] [INSPIRE].

[30] C. Schwinn and S. Weinzierl, SUSY Ward identities for multi-gluon helicity amplitudes with massive quarks, JHEP 03 (2006) 030 [hep-th/0602012] [INSPIRE].

[31] N. Craig, H. Elvang, M. Kiermaier and T. Slatyer, Massive amplitudes on the Coulomb branch of $N=4$ SYM, JHEP 12 (2011) 097 [arXiv:1104.2050] [INSPIRE].

[32] R.H. Boels and C. Schwinn, On-shell supersymmetry for massive multiplets, Phys. Rev. D 84 (2011) 065006 [arXiv: 1104.2280] [INSPIRE].

[33] A. Guevara, Holomorphic classical limit for spin effects in gravitational and electromagnetic scattering, arXiv:1706.02314 [INSPIRE].

[34] N. Moynihan and J. Murugan, Comments on scattering in massive gravity, vDVZ and $B C F W$, arXiv: 1711.03956 [INSPIRE].

[35] N. Christensen and B. Field, The constructive Standard Model: part I, arXiv:1802.00448 [INSPIRE].

[36] E. Conde and A. Marzolla, Lorentz constraints on massive three-point amplitudes, JHEP 09 (2016) 041 [arXiv: 1601.08113] [INSPIRE].

[37] E. Conde, E. Joung and K. Mkrtchyan, Spinor-helicity three-point amplitudes from local cubic interactions, JHEP 08 (2016) 040 [arXiv: 1605.07402] [INSPIRE].

[38] L.M. Brown, Two-component Fermion theory, Phys. Rev. 111 (1958) 957 [InSPIRE].

[39] M. Tonin, Quantization of the two-component fermion theory, Nuovo Cim. 14 (1959) 1108.

[40] G. Chalmers and W. Siegel, Simplifying algebra in Feynman graphs, part I: spinors, Phys. Rev. D 59 (1999) 045012 [hep-ph/9708251] [INSPIRE].

[41] G. Chalmers and W. Siegel, Simplifying algebra in Feynman graphs, part II: spinor helicity from the space-cone, Phys. Rev. D 59 (1999) 045013 [hep-ph/9801220] [INSPIRE]. 
[42] G. Chalmers and W. Siegel, Simplifying algebra in Feynman graphs, part III: massive vectors, Phys. Rev. D 63 (2001) 125027 [hep-th/0101025] [INSPIRE].

[43] S.D. Badger, E.W.N. Glover, V.V. Khoze and P. Svrček, Recursion relations for gauge theory amplitudes with massive particles, JHEP 07 (2005) 025 [hep-th/0504159] [INSPIRE].

[44] S.D. Badger, E.W.N. Glover and V.V. Khoze, Recursion relations for gauge theory amplitudes with massive vector bosons and fermions, JHEP 01 (2006) 066 [hep-th/0507161] [INSPIRE].

[45] K.J. Ozeren and W.J. Stirling, Scattering amplitudes with massive fermions using BCFW recursion, Eur. Phys. J. C 48 (2006) 159 [hep-ph/0603071] [INSPIRE].

[46] J.-H. Huang and W. Wang, Multigluon tree amplitudes with a pair of massive fermions, Eur. Phys. J. C 72 (2012) 2050 [arXiv: 1204.0068] [inSPIRE].

[47] J.M. Drummond and J.M. Henn, All tree-level amplitudes in N=4 SYM, JHEP 04 (2009) 018 [arXiv: 0808.2475] [INSPIRE].

[48] L.J. Dixon, J.M. Henn, J. Plefka and T. Schuster, All tree-level amplitudes in massless QCD, JHEP 01 (2011) 035 [arXiv: 1010.3991] [INSPIRE].

[49] Z. Bern, L.J. Dixon, D.C. Dunbar and D.A. Kosower, One loop $n$ point gauge theory amplitudes, unitarity and collinear limits, Nucl. Phys. B 425 (1994) 217 [hep-ph/9403226] [INSPIRE].

[50] Z. Bern, L.J. Dixon, D.C. Dunbar and D.A. Kosower, Fusing gauge theory tree amplitudes into loop amplitudes, Nucl. Phys. B 435 (1995) 59 [hep-ph/9409265] [INSPIRE].

[51] R. Britto, F. Cachazo and B. Feng, Generalized unitarity and one-loop amplitudes in $N=4$ super-Yang-Mills, Nucl. Phys. B 725 (2005) 275 [hep-th/0412103] [INSPIRE].

[52] D. Forde, Direct extraction of one-loop integral coefficients, Phys. Rev. D 75 (2007) 125019 [arXiv: 0704.1835] [INSPIRE].

[53] R. Britto, E. Buchbinder, F. Cachazo and B. Feng, One-loop amplitudes of gluons in SQCD, Phys. Rev. D 72 (2005) 065012 [hep-ph/0503132] [INSPIRE].

[54] C. Anastasiou, R. Britto, B. Feng, Z. Kunszt and P. Mastrolia, D-dimensional unitarity cut method, Phys. Lett. B 645 (2007) 213 [hep-ph/0609191] [InSPIRE].

[55] W.T. Giele, Z. Kunszt and K. Melnikov, Full one-loop amplitudes from tree amplitudes, JHEP 04 (2008) 049 [arXiv: 0801.2237] [INSPIRE].

[56] S. Badger, C. Bronnum-Hansen, F. Buciuni and D. O'Connell, A unitarity compatible approach to one-loop amplitudes with massive fermions, JHEP 06 (2017) 141 [arXiv: 1703.05734] [INSPIRE].

[57] C. Cheung and D. O'Connell, Amplitudes and spinor-helicity in six dimensions, JHEP 07 (2009) 075 [arXiv:0902.0981] [INSPIRE].

[58] M. Dinsdale, M. Ternick and S. Weinzierl, A comparison of efficient methods for the computation of Born gluon amplitudes, JHEP 03 (2006) 056 [hep-ph/0602204] [INSPIRE].

[59] S. Badger, B. Biedermann, L. Hackl, J. Plefka, T. Schuster and P. Uwer, Comparing efficient computation methods for massless QCD tree amplitudes: closed analytic formulas versus Berends-Giele recursion, Phys. Rev. D 87 (2013) 034011 [arXiv:1206.2381] [INSPIRE].

[60] D. Maître and P. Mastrolia, S@M, a mathematica implementation of the spinor-helicity formalism, Comput. Phys. Commun. 179 (2008) 501 [arXiv:0710.5559] [INSPIRE]. 